\title{
GEOMETRIC AND NUMERICAL TECHNIQUES IN OPTIMAL CONTROL OF TWO AND THREE-BODY PROBLEMS*
}

\author{
B. BONNARD ${ }^{\dagger}$, J.-B. CAILLAU ${ }^{\ddagger}$, AND G. PICOT $^{\S}$
}

\begin{abstract}
The objective of this article is to present geometric and numerical techniques developed to study the orbit transfer between Keplerian elliptic orbits in the two-body problem or between quasi-Keplerian orbits in the Earth-Moon transfer when low propulsion is used. We concentrate our study on the energy minimization problem. From Pontryagin's maximum principle, the optimal solution can be found solving the shooting equation for smooth Hamiltonian dynamics. A first step in the analysis is to find in the Kepler case an analytical solution for the averaged Hamiltonian, which corresponds to a Riemannian metric. This will allow to compute the solution for the original Kepler problem, using a numerical continuation method where the smoothness of the path is related to the conjugate point condition. Similarly, the solution of the Earth-Moon transfer is computed using geometric and numerical continuation techniques.
\end{abstract}

1. Introduction. In this article we consider the orbit transfer in the two and three-body problem, using low propulsion. In the first case, the model is given by Kepler equation

$$
\ddot{q}=-\frac{q}{|q|^{3}}+\frac{u}{m}
$$

where $m$ represents the mass of the satellite, subject to

$$
\dot{m}=-\delta|u|
$$

modelling fuel consumption. The control satisfies the constraint $|u| \leq \varepsilon$ where $\varepsilon$ is a small parameter.

The physical optimal control has to maximize the final mass which leads to

$$
\min _{u(.)} \int_{0}^{t_{f}}|u| \mathrm{d} t
$$

where $t_{f}$ is the fixed transfer time. From Pontryagin maximum principle [28], fixing the boundary conditions - e.g. a transfer from a low eccentric to a geostationary orbit-, an optimal solution can be numerically computed using a shooting algorithm.

\footnotetext{
*Dedicated to John Baillieul on the Occasion of His 65th Birthday.

${ }^{\dagger}$ Mathematics Institute, Bourgogne University \& CNRS, 9 avenue Savary, F-21078 Dijon. E-mail: bernard.bonnard@u-bourgogne.fr

${ }^{\ddagger}$ Mathematics Institute, Bourgogne University \& CNRS, 9 avenue Savary, F-21078 Dijon. Email: jean-baptiste.caillau@u-bourgogne.fr, supported by Conseil Régional de Bourgogne (contract no. 2009-160E-160-CE-160T).

$\S$ Mathematics Institute, Bourgogne University \& CNRS, 9 avenue Savary, F-21078 Dijon. E-mail: gautier.picot@u-bourgogne.fr, supported by CNRS (contract no. 37244) and Conseil Régional de Bourgogne (contract no. 079201PP02454515).
} 
This leads to a complicated numerical problem. In [20], the following numerical scheme is proposed. One computes the optimal solution using the convex homotopy

$$
\min _{u(.)} \int_{0}^{t_{f}}\left[\lambda|u|^{2}+(1-\lambda)|u|\right] \mathrm{d} t, \quad \lambda \in[0,1]
$$

which amounts to regularizing the $\mathrm{L}^{1}$-minimization problem into an $\mathrm{L}^{2}$-problem. This was the starting point of the use of continuation methods in orbital transfer, when low propulsion is applied - see also [14] for the use of the continuation method in the time minimal control problem, where the homotopy parameter is the bound of the maximal amplitude of the thrust. From the mathematical point of view, the original dynamics associated with the optimal flow is replaced by another Hamiltonian one, and a continuation is made to solve the shooting equation.

The first motivation of this article is to present a neat geometric result from [8]: Neglecting the mass variation, restricting to coplanar transfer (the inclination being considered as a homotopy parameter) and replacing the $\mathrm{L}^{1}$-problem by an averaged $\mathrm{L}^{2}$-problem, one can substitute the Hamiltonian vector field defined by the maximum principle with

$$
H=\frac{1}{2 n^{5 / 3}}\left[9 n^{2} p_{n}^{2}+\frac{5}{2}\left(1-e^{2}\right) p_{e}^{2}+\frac{5-4 e^{2}}{2} \frac{p_{\theta}^{2}}{e^{2}}\right],
$$

where $n$ is the mean motion, $e$ the eccentricity, and $\theta$ the angle of the pericenter (the singularity $e=0$ corresponds to circular orbits). Coordinates $(n, e, \theta)$ are moreover orthogonal coordinates for the Riemannian metric associated with $H$,

$$
g=\frac{\mathrm{d} n^{2}}{9 n^{\frac{1}{3}}}+\frac{2 n^{5 / 3}}{5\left(1-e^{2}\right)} \mathrm{d} e^{2}+\frac{2 n^{5 / 3}}{5-4 e^{2}} \mathrm{~d} \theta^{2} .
$$

Such a metric is isometric to

$$
g=\mathrm{d} r^{2}+\frac{r^{2}}{c^{2}}\left(\mathrm{~d} \varphi^{2}+G(\varphi) \mathrm{d} \theta^{2}\right)
$$

where

$$
r=\frac{2}{5} n^{5 / 6}, \quad \varphi=\arcsin e
$$

and

$$
c=\sqrt{2 / 5}, \quad G(\varphi)=\frac{5 \sin ^{2}(\varphi)}{1+4 \cos ^{2}(\varphi)} .
$$

The Hamiltonian flow $\vec{H}$ is Liouville integrable and the metric in the above normal form captures the main properties of the averaged orbital transfer. Indeed, one can extract from $g$ the following two-dimensional Riemannian metrics:

- $g_{1}=\mathrm{d} r^{2}+r^{2} \mathrm{~d} \psi^{2}$ which is associated with the orbital transfer where $\theta$ is kept fixed (this encompasses the case of circular targets). Such a metric is flat and geodesics are straight lines in suitable coordinates. 
- $g_{2}=\mathrm{d} \varphi^{2}+G(\varphi) \mathrm{d} \theta^{2}$ which represents the restriction of the metric to $r^{2}=c^{2}$ and describes by homogeneity the orbit transfer in the general case.

A generalization of the results of [31] will allow to compute for the metric $g_{2}$ the conjugate and cut loci and to get a global optimality solution for the averaged optimal control problem. This is the starting point to analyze the original optimal control problem using a continuation method.

A second motivation of this article is to present some results from geometric control theory connected to our analysis with adapted numerical codes developed to compute the solutions. First of all, the maximum principle is only a necessary optimality condition. In order to get sufficient optimality conditions under generic assumptions one must define the concept of conjugate point, associated with the energy minimization problem. This concept was already introduced in the standard litterature of calculus of variations [5]. If the Hamiltonian optimal dynamics is described by a smooth Hamiltonian vector field $\vec{H}$, conjugate points are the image of the singularities of the exponential mapping: $\exp _{x(0)}: p(0) \longrightarrow \Pi_{x} \exp t_{f} \vec{H}(x(0), p(0))$ where $\Pi_{x}:(x, p) \longrightarrow x$ is the canonical projection. Such points can be numerically computed using the code [12]. An important remark, in view of the use of the (smooth) continuation method in optimal control is to observe that the shooting equation is precisely to find $p(0)$ such that $\exp _{x(0)}(p(0))=x_{1}$ where $x_{1}$ is the terminal condition and the derivative is generated using the variational equation of $\vec{H}$. This will lead to convergence results for the smooth continuation method in optimal control, related to estimates of conjugate points.

The last section is devoted to the Earth-Moon transfer, using low propulsion. The model is the standard circular restricted model [25] where the two primaries are fixed in a rotating frame. Up to a normalization the system can be written in Hamiltonian form,

$$
\dot{x}=\overrightarrow{H_{0}}(x)+u_{1} \overrightarrow{H_{1}}(x)+u_{2} \overrightarrow{H_{2}}(x)
$$

where $x=(q, p) \in \mathbf{R}^{4}$ and the drift $\overrightarrow{H_{0}}$ is given by

$$
H_{0}(x)=\frac{1}{2}\left(p_{1}^{2}+p_{2}^{2}\right)+p_{1} q_{2}-p_{2} q_{1}-\frac{1-\mu}{\rho_{1}}-\frac{\mu}{\rho_{2}},
$$

$q$ being the position of the spacecraft, $\rho_{1}$ representing the distance to the Earth with mass $1-\mu$ located at $(-\mu, 0)$, and $\rho_{2}$ the distance to the Moon with mass $\mu$ located at $(1-\mu, 0), \mu \simeq 1.2153 e-2$ being a small parameter. The Hamiltonian fields associated with the control are given by

$$
H_{i}(x)=-q_{i}, \quad i=1,2,
$$

and the control bound is $|u| \leq \varepsilon$. The parameter $\mu$ is small and this remark was used by Poincare to study the dynamics of the free motion described by $\overrightarrow{H_{0}}$ by making 
a deformation of the case $\mu=0$ which corresponds to Kepler equation in rotating coordinates [25]. Inspired by this approach and using our preliminary geometric analysis, we propose a simple solution to the Earth-Moon transfer using low propulsion for the energy minimization problem.

\section{Geometric and numerical methods.}

2.1. Maximum principle. We consider the energy minimization problem

$$
\min _{u(.)} \int_{0}^{t_{f}}|u|^{2} \mathrm{~d} t
$$

for a smooth control system of the form

$$
\dot{x}=F_{0}(x(t))+\sum_{i=1}^{m} u_{i}(t) F_{i}(x(t))=F(x(t), u(t)), \quad x \in X .
$$

The set of admissible controls is the subset $\mathcal{U}$ of measurable bounded mappings $u($. with corresponding trajectory $x($.$) defined on the whole interval \left[0, t_{f}\right]$. Pontryagin maximum principle [28] tells us that

Proposition 2.1. If $(x, u)$ is an optimal pair on $\left[0, t_{f}\right]$, there exists a non trivial pair $\left(p^{0}, p\right), p^{0} \leq 0$ and $p$ an absolutely continuous adjoint vector valued in $T^{*} X$, such that on $\left[0, t_{f}\right]$ we have

$$
\dot{x}=\frac{\partial H}{\partial p}(x, p, u), \quad \dot{p}=-\frac{\partial H}{\partial x}(x, p, u),
$$

and

$$
H(x, p, u)=\max _{v \in \mathbf{R}^{m}} H(x, p, v)
$$

where $H(x, p, u)=p^{0} \sum_{i=1}^{m} u_{i}^{2}+<p, F(x, u)>$.

Definition 2.1. The mapping $H$ from $T^{*} X \times \mathbf{R}^{m}$ to $\mathbf{R}$ is called the pseudoHamiltonian. A triple $(x, p, u)$ solution of (1-2) is called an extremal trajectory.

2.2. Computation of extremals. From the maximization condition (4), one deduces that $\partial H / \partial v=0$, and there are two types of extremals:

- Abnormal extremals. They correspond to the situation $p^{0}=0$ and are implicitely defined by the relations $H_{i}=0, i=1, \ldots, m$, where $H_{i}=<$ $p, F_{i}(x)>$ are the Hamiltonian lifts.

- Normal extremals. If $p^{0}<0$, it can be normalized to $-1 / 2$ by homogeneity. From $\partial H / \partial v=0$, one deduces $u_{i}=H_{i}$ for $i=1, \ldots, m$, and plugging such $H_{i}$ into $H$ defines a true smooth Hamiltonian

$$
H_{n}=H_{0}+\frac{1}{2} \sum_{i=1}^{m} H_{i}^{2}
$$

whose solutions are the normal extremals. 


\subsection{The concept of conjugate point.}

Definition 2.2. Let $z=(x, p)$ be a normal reference extremal defined on $\left[0, t_{f}\right]$. The variational equation

$$
\dot{\delta} z(t)=\mathrm{d} \overrightarrow{H_{n}}(z(t)) \delta z(t)
$$

is called the Jacobi equation. A Jacobi field is a non-trivial solution $\delta z=(\delta x, \delta p)$. It is said to be vertical at time $t$ if $\delta x(t)=\mathrm{d} \Pi_{x}(z(t)) \delta z(t)=0$ where $\Pi_{x}$ is the projection $(x, p) \mapsto x$.

The following standard geometric result is crucial.

Proposition 2.2. Let $L_{0}$ be the fiber $T_{x_{0}}^{*} X$ and $L_{t}=\exp _{t}\left(\overrightarrow{H_{n}}\right)\left(L_{0}\right)$ be its image by the one-parameter subgroup generated by $\vec{H}_{n}$. Then $L_{t}$ is a Lagrangian submanifold whose tangent space at $z(t)$ is generated by the Jacobi fields which are vertical at $t=0$.

Definition 2.3. We fix $x_{0}=x(0)$ and define for $t \in\left[0, t_{f}\right]$ the exponential mapping

$$
\exp _{x_{0}, t}\left(p_{0}\right)=\Pi_{x}\left(z\left(t, z_{0}\right)\right)
$$

where $z\left(t, z_{0}\right)$, with $z_{0}=\left(x_{0}, p_{0}\right)$, denotes the normal extremal departing from $z_{0}$ when $t=0$.

Definition 2.4. Let $z=(x, p)$ be the reference normal extremal. The time $t_{c} \in\left[0, t_{f}\right]$ is called conjugate if the mapping $\exp _{x_{0}, t_{c}}$ is not an immersion at $p(0)$. The associated point $x\left(t_{c}\right)$ is said to be conjugate to $x_{0}$. We denote by $t_{1, c}$ the first conjugate point and by $C\left(x_{0}\right)$ the conjugate locus formed by the set of first conjugate points occuring at time $t_{f}$ when we consider all normal extremals starting from $x_{0}$.

The conjugate time notion admits the following generalization.

Definition 2.5. Let $M_{1}$ be a regular submanifold of $M$, and let us define $M_{1}^{\perp}=$ $\left\{(x, p) \in T^{*} M \mid x \in M_{1}, p \perp T_{x} M_{1}\right\}$. Then $t_{\mathrm{foc}} \in\left[0, t_{f}\right]$ is called a focal time if there exists a Jacobi field $J=(\delta x, \delta p)$ such that $\delta x(0)=0$ and $J\left(t_{\text {foc }}\right)$ is tangent to $M_{1}^{\perp}$.

REMARK 2.1. The concept of conjugate point is related to the necessary and sufficient optimality conditions, under generic assumptions, see for instance [12].

2.4. Conjugate points and smooth continuation method. Smooth continuation is a general numerical method to solve a system of equations $F(x)=0$ where $F: \mathbf{R}^{n} \longrightarrow \mathbf{R}^{n}$ is a smooth mapping, see [1]. The principle is to construct a homotopy path $h(x, \lambda)$ such that $h(x, 0)=G(x)$ and $h(x, 1)=F(x)$ where $G(x)$ is a map having known zeros, or where the zeros can be easily computed using a Newton type algorithm. The zeros along the path can be calculated by different methods, the simplest being a discretization $0=\lambda_{0}<\lambda_{1}<\cdots<\lambda_{n}=1$ of the homotopy parameter where, at step $i+1$, the zero computed at step $i$ is used to initialize Newton algorithm. The approach has to be adapted to optimal control problems: The shooting equation comes from the projection of a symplectic mapping, the Jacobian can be computed 
using Jacobi fields and one must consider the central extremal fields associated with the problem (see [13]). A short description of the method is given below in our case study.

2.4.1. Shooting equation. We consider a family $H_{\lambda}, \lambda \in[0,1]$, of smooth Hamiltonians on $T^{*} X$ associated with normal extremals of an energy minimization problem. We fix the boundary conditions $x_{0}, x_{1}$ and the transfer time $t_{f}$. This leads to a family $\exp _{x_{0}, t_{f}}^{\lambda}\left(p_{0}\right)$ of exponential mappings. Using the notation $E^{\lambda}: p_{0} \longrightarrow$ $\exp _{x_{0}, t_{f}}^{\lambda}\left(p_{0}\right)$, one must solve the shooting equation $E^{\lambda}\left(p_{0}\right)=x_{1}$.

Proposition 2.3. For each $\lambda$, the shooting equation is of maximal rank if and only if the point $x_{1}$ is not conjugate to $x_{0}$ for the corresponding $\lambda$. Moreover, in this case, the solutions of the shooting equation contain a smooth branch, which can be parameterized by $\lambda$ and the derivative $E^{\prime \lambda}$ can be generated integrating the Jacobi equation.

From the above proposition, to ensure convergence of the method one must control

- the distance to the conjugate loci,

- that the branch has is defined on the whole interval $[0,1]$.

The second point is related to two standard problems in optimal control: Existence of Lipschitzian minimizers - hence solutions of the maximum principle [22] - , and compactness of the domain of the exponential mapping. Next we present a nice geometric situation for which convergence of the method is ensured.

Definition 2.6. Consider the normal extremal field $\overrightarrow{H_{n}}$ of an energy minimization problem with fixed final time $t_{f}$. Given an initial condition $x_{0}$, the separating locus $L\left(x_{0}\right)$ is the set of points where two distinct normal extremal curves intersect with same cost. The cut point along a normal extremal is the first point where it ceases to be optimal. The cut locus $\operatorname{Cut}\left(x_{0}\right)$ is the set of such points when we consider all extremals initiating from $x_{0}$ and losing optimality exactly at time $t_{f}$.

\subsubsection{Convergence of the continuation method in the Riemannian case.}

We first recall that the Riemannian problem can be, at least locally, reset in the following framework.

Let $F_{1}, \ldots, F_{n}$ be a set of smooth vector fields on a manifold $\mathrm{X}$ and assume that they are linearly independent. One can define a Riemannian metric on $X$ by asserting that $\left\{F_{1}, \ldots, F_{n}\right\}$ form an orthonormal frame. Introducing the control system $\mathrm{d} x(t) / \mathrm{d} t=\sum_{i=1}^{n} u_{i}(t) F_{i}(x(t))$, the length of the curve $x(\cdot)$ is $l(x)=$ $\int_{0}^{T} \sum_{i=1}^{n}\left(u_{i}^{2}(t)\right)^{1 / 2} \mathrm{~d} t$. From Maupertuis principle, minimizing length is equivalent to minimizing the energy $\int_{0}^{T} \sum_{i=1}^{n} u_{i}^{2}(t) \mathrm{d} t$. There exists only normal extremals and $H_{n}$ is given by $(1 / 2) \sum_{i=1}^{n} H_{i}^{2}$. Fixing the level set $H_{n}=1 / 2$ parameterizes trajectories by arc length. For the energy minimization problem, the transfer time can be arbitrarily prescribed.

TheOREM 2.1. Let $g_{\lambda}, \lambda \in[0,1]$, be a smooth family of complete Riemannian 
metrics on $X$. Let us fix the initial point $x_{0}$. Denote $i_{\lambda}\left(x_{0}\right)$ the distance from $x_{0}$ to the cut locus $\operatorname{Cut}^{\lambda}\left(x_{0}\right)$ and by $i_{\lambda}=\inf _{x_{0}} i_{\lambda}\left(x_{0}\right)$ the injectivity radius of the corresponding metric. Then,

- for length shorter than $\inf _{\lambda} i_{\lambda}\left(x_{0}\right)$, the continuation method with initial condition of the shooting equation at $x_{0}$ converges,

- for length shorter than $\inf _{\lambda} i_{\lambda}$ the continuation method converges for every initial condition of the shooting equation.

Remark 2.2. In the Riemannian case, the situation is neat. Completeness leads to existence of smooth normal minimizers, the domain of the exponential mapping is a sphere, and estimates of the injectivity radius are related to the curvature tensor. In general, such estimates are a difficult problem and a pragmatic point of view is to have numerical approximations [12].

\section{The energy minimization problem in orbital transfer with low} thrust.

3.1. Preliminaries. Neglecting the mass variation and restricting to the coplanar case, the system is represented in Cartesian coordinates by

$$
\ddot{q}=-\frac{q}{|q|^{3}}+u
$$

where $q=\left(q_{1}, q_{2}\right)$ is the position and $x=(q, \dot{q}) \in \mathbf{R}^{4}$ is the state. We denote by $H_{0}(q, \dot{q})=(1 / 2) \dot{q}^{2}-1 /|q|$ the Hamiltonian of the free motion. We have the following first integrals:

- $C=q \wedge \dot{q}$ (momentum),

- $\mathrm{L}=-q /|q|+\dot{q} \wedge C$ (Laplace integral).

Proposition 3.1. The domain $\Sigma_{e}=\{(q, \dot{q}) \mid H<0, C \neq 0\}$, called the elliptic domain, is filled by elliptic orbits and to each orbit $(C, L)$ corresponds a unique (oriented) ellipse.

To represent the space of ellipses, one introduces the following geometric coordinates:

- the semi-major axis of the ellipse $a$, related to the semi-latus rectum $P$ by the relation $a=P / \sqrt{1-e^{2}}$,

- the argument of the pericenter $\theta$,

- the eccentricity $e$, the eccentricity vector being $\left(e_{x}, e_{y}\right)=(e \cos \theta, e \sin \theta)$.

To represent the position of the satellite we use the longitude $l \in \mathbf{S}^{1}$, while $l \in \mathbf{R}$ takes into account the rotation number and is called the cumulated longitude. Observe that $e=0$ corresponds to circular orbits. The control $u$ can be decomposed into moving frames attached to the satellite, the two standard frames being

- the radial-orthoradial frame $\left\{F_{r}, F_{o r}\right\}$ where $F_{r}=(q /|q|) \partial / \partial \dot{q}$,

- the tangential-normal frame $\left\{F_{t}, F_{n}\right\}$ where $F_{t}=(\dot{q} /|\dot{q}|) \partial / \partial \dot{q}$. 
3.2. Averaging of periodic sub-Riemannian problems. Let $X$ be an $n$ dimensional smooth manifold and let $F_{i}(l, x), i=1, \ldots, m$ be smooth vector fields parameterized by $l \in \mathbf{S}^{1}$ that set up a constant rank $m$ distribution on $X$ (see also Remark 3.2). Given a positive pulsation $\omega$ on $\mathbf{S}^{1} \times X$ relating the time $t$ and the angle $l$ according to

$$
\mathrm{d} l=\omega(l, x) \mathrm{d} t,
$$

one defines a periodic sub-Riemannianproblem as follows: Given two points $x_{0}$ and $x_{f}$ on the manifold, minimize the $\mathrm{L}^{2} \mathrm{~d} t$ control norm of trajectories connecting the two points and associated with the previous vector fields,

$$
\begin{gathered}
\frac{\mathrm{d} x}{\mathrm{~d} l}=\sum_{i=1}^{m} u_{i} F_{i}(l, x), \quad u \in \mathbf{R}^{m}, \\
\min _{u(.)} \int_{0}^{t_{f}}|u|^{2} \mathrm{~d} t=\int_{0}^{l_{f}}|u|^{2} \frac{\mathrm{d} l}{\omega(l, x)} \quad\left(\text { here }|u|^{2}=\sum_{i=1}^{m}\left|u_{i}\right|^{2}\right) .
\end{gathered}
$$

The total angular length, $l_{f}>0$ is fixed, implicitly defining $t_{f}$ through (3).

Pontryagin maximum principle asserts that minimizing trajectories are projection of Hamiltonian curves on the cotangent bundle (extremals), $z=(x, p)$, such that

$$
\frac{\mathrm{d} x}{\mathrm{~d} l}=\frac{\partial H}{\partial p}(l, x, p, u), \quad \frac{\mathrm{d} p}{\mathrm{~d} l}=-\frac{\partial H}{\partial x}(l, x, p, u),
$$

where

$$
H(l, x, p, u)=p^{0}|u|^{2}+\sum_{i=1}^{m} u_{i} H_{i}(l, x, p), \quad H_{i}=\left\langle p, F_{i}(l, x)\right\rangle, \quad i=1, \ldots, m,
$$

is the Hamiltonian parameterized by $l \in \mathbf{S}^{1}, u \in \mathbf{R}^{m}$, and a non-positive constant $p^{0}$. We restrict the analysis to the normal case, $p^{0}<0$, and pass to affine coordinates in $\left(p^{0}, p\right)$ setting $p^{0}=-1 / 2$. Pontryagin maximization condition that, almost everywhere along an extremal,

$$
H(l, z(l), u(l))=\max _{v \in \mathbf{R}^{m}} H(l, z(l), v),
$$

implies that the control is given by the dynamic feedback

$$
u(l, z)=\omega(l, x)\left(H_{1}, \ldots, H_{m}\right)(l, z),
$$

and that $z$ is an integral curve of the maximized Hamiltonian

$$
H_{n}(l, z)=\frac{\omega(l, x)}{2} \sum_{i=1}^{m} H_{i}^{2}(l, z) .
$$


We are interested in the behaviour of solutions for large angular length, so we set $\varepsilon=$ $1 / l_{f}$ as the small parameter and renormalize the problem in the following mannertypical of systems with two time scales.

In contrast to $l$ which is the fast time, define the slow time $s=\varepsilon l$ in $[0,1]$, and renormalize variables on the cotangent bundle by

$$
\widetilde{x}=x, \quad \widetilde{p}=p / \varepsilon .
$$

LEMMA 3.1. In the renormalized variables,

$$
\frac{\mathrm{d} \widetilde{z}}{\mathrm{~d} s}=\vec{H}_{n}(s / \varepsilon, \widetilde{z}) .
$$

Proof. Obvious since $H_{n}(l, x, \cdot)$ is quadratic in the adjoint state $p$.

For fixed $z, H_{n}(\cdot, z)$ is a smooth function on $\mathbf{S}^{1}$ and can be expanded into its Fourier series. In particular, one can define its first coefficient or average,

$$
H(z)=\frac{1}{2 \pi} \int_{0}^{2 \pi} H_{n}(l, z) \mathrm{d} l .
$$

Since taking symplectic vector field and averaging readily commute, it is well known $[2,21]$ that $H$ trajectories are good approximations of those of $H_{n}$ as $\varepsilon$ goes to zero.

THEOREM 3.1. Given any initial condition, the solution $\widetilde{z}_{\varepsilon}$ of (5) converges uniformly on $[0,1]$ towards the solution $\bar{z}$ of the averaged Hamiltonian.

REMARK 3.1. The same rate of convergence holds for the cost which can be added as a new state in the augmented system. But as the integrand depends on the fast variable $l$ through the pulsation $\omega$, there is a priori no higher order approximation of the performance index [4, 16].

As for $H_{n}(s / \varepsilon, z)$ which converges to $H$ in the large space of Schwartz distributions, ${ }^{1}$ one can only expect weak convergence on the control $u_{\varepsilon}$ of the original problem on $[0,1 / \varepsilon]$. We address two questions: First, can we describe the fast oscillating control by means of Fourier series with slowly varying coefficients and, secondly, what is the asymptotic behaviour of $\left\|u_{\varepsilon}\right\|_{\infty}$ when $\varepsilon \rightarrow 0$ ? The importance of such an estimate relies on the fact that one has in practice to estimate $l_{f}$ so as to meet a given requirement on the $\mathrm{L}^{\infty}$-norm of the control.

We first note that the average system provides a sub-Riemannian approximation of the original one.

Proposition 3.2. The averaged Hamiltonian is a nonnegative quadratic form in the adjoint. If constant, its rank is not less than $m$, and $H$ can locally be written as a sum of squares, thus defining a sub-Riemannian (Riemannian if $k=n$ ) problem.

Proof. Clearly, $H(x, \cdot)$ remains quadratic nonnegative in $p$ by linearity and positivity of the integral and

$$
\operatorname{ker} H(x, \cdot)=\bigcap_{l \in \mathbf{S}^{1}} \operatorname{ker} H_{n}(l, x, \cdot)
$$

\footnotetext{
${ }^{1}$ Topological dual of the space of smooth compactly supported functions on the real line.
} 
so that, if constant, the rank $k$ is at least equal to $m$. In this case, the quadratic form can always be decomposed into a sum of $k$ squares in a chart by taking the square root of the associated symmetric nonnegative matrix.

REMARK 3.2. (i) The increase in the rank is related to the generation of Lie brackets of the following system with drift: Set $\hat{x}=(l, x)$ and define

$$
\hat{F}_{0}(\hat{x})=\omega(\hat{x}) \frac{\partial}{\partial l}, \quad \hat{F}_{i}(\hat{x})=\omega(\hat{x}) F_{i}(\hat{x}), \quad i=1, \ldots, m .
$$

The fast oscillations (with respect to l) of the control in

$$
\frac{\mathrm{d} \hat{x}}{\mathrm{~d} t}=\hat{F}_{0}(\hat{x})+\sum_{i=1}^{m} u_{i} \hat{F}_{i}(\hat{x})
$$

generate new directions, namely

$$
\left(\operatorname{ad}^{j} \hat{F}_{0}\right) \hat{F}_{i}, \quad j \geq 0, i=1, \ldots, m,
$$

a natural requirement being that the distribution $\left\{\hat{F}_{0}, \hat{F}_{1}, \ldots, \hat{F}_{m}\right\}$ on the augmented space $\mathbf{S}^{1} \times X$ be bracket generating. This is equivalent to the bracket condition on $\mathbf{S}^{1} \times X$ for the distribution $\left\{\partial / \partial l, F_{1}, \ldots, F_{m}\right\}$.

(ii) The assumption of rank constancy is sufficient to get a decomposition into a smooth sum of squares [3]. That the assumption is crucial is illustrated by the fact that it cannot be removed, even in the analytical category. Eigenvalues (and associated projectors) are indeed analytic functions on the set of matrices in the neighbourhoud of a simple (hence diagonalizable) endomorphism [23, Theorem II.5.16]. Avoiding semi-simple eigenvalues is necessary as is clear considering

$$
\left[\begin{array}{rr}
x_{1} & x_{2} \\
x_{2} & -x_{1}
\end{array}\right]
$$

whose eigenvalues are not differentiable at $(0,0)$. But even in the simple symmetric nonnegative analytic case, existence of a differentiable square root matrix may fail as illustrated by

$$
\left[\begin{array}{cc}
x_{1}^{2}+x_{2}^{2} & 0 \\
0 & 1
\end{array}\right] .
$$

To get a positive result with non constant rank, one must actually restrict to the symmetric nonnegative case with analytic dependence on one real variable only. Eigenvalues and eigenvectors are then analytic on the real line [Ibid., Theorem II.6.1 and $\S I I .6 .2]$, and nonnegativeness ensures analyticity of square roots of the eigenvalues.

(iii) In the case of periodic sub-Riemannian systems, the loss of regularity may originate in averaging. The analytical distribution on $\mathbf{S}^{1} \times \mathbf{R}^{2}$

$$
F_{1}(l, x)=\sqrt{2}\left(x_{1} \cos l+x_{2} \sin l\right) \frac{\partial}{\partial x_{1}}, \quad F_{2}(l, x)=\frac{\partial}{\partial x_{2}}
$$


has quadratic form

$$
\left[\begin{array}{cc}
2\left(x_{1} \cos l+x_{2} \sin l\right)^{2} & 0 \\
0 & 1
\end{array}\right]
$$

whose averaged is (6) which does not even define a differentiable sub-Riemannian system on $\mathbf{R}^{2}$.

(iv) A much stronger requirement is the existence of a change of coordinates on $X$ (inducing a symplectic transformation on the cotangent) so that the averaged quadratic form be diagonal.

For a given $z \in T^{*} X$, let

$$
u(l, z)=\sum_{k \in \mathbf{Z}} c_{k}(z) e_{k}(l), \quad e_{k}(l)=e^{i k l},
$$

denote the Fourier series of the control (4). One has the following convergence result.

Proposition 3.3. For any positive $\varepsilon$, the normal optimal control is

$$
u_{\varepsilon}(l)=\varepsilon \sum_{k \in \mathbf{Z}} c_{k} \circ \widetilde{z}_{\varepsilon}(\varepsilon l) e_{k}(l)
$$

and the series converges pointwisely. Moreover, for any $k \in \mathbf{Z}$,

$$
c_{k} \circ \widetilde{z}_{\varepsilon} \rightarrow c_{k} \circ \bar{z} \text { uniformly on }[0,1] \text { as } \varepsilon \rightarrow 0+
$$

where the sub-Riemannian extremal $\bar{z}$ depends only on the boundary conditions $x_{0}$, $x_{f}$ on $X$.

Proof. For $l \in\left[0, l_{f}\right]$,

$$
\begin{aligned}
u_{\varepsilon}(l) & =u\left(l, \widetilde{x}_{\varepsilon}(\varepsilon l), \varepsilon \widetilde{p}_{\varepsilon}(\varepsilon l)\right) \\
& =\varepsilon u\left(l, \widetilde{x}_{\varepsilon}(\varepsilon l), \widetilde{p}_{\varepsilon}(\varepsilon l)\right) \\
& =\varepsilon \sum_{k \in \mathbf{Z}} c_{k}\left(\widetilde{z}_{\varepsilon}(\varepsilon l)\right) e_{k}(l)
\end{aligned}
$$

thanks to the pointwise convergence of the Fourier series at $l$ for $z=\widetilde{z}_{\varepsilon}(l)$. For any $k \in \mathbf{Z}$,

$$
c_{k}(z)=\frac{1}{2 \pi} \int_{0}^{2 \pi} u(l, z) \bar{e}_{k}(l) \mathrm{d} l
$$

and the dependence on $z$ is continuous since the integrand is bounded in a compact neighbourhood of the image of $\bar{z}$.

Let us finally denote $\lambda(l, x)$ the biggest (positive) eigenvalue of the nonnegative quadratic form in $p$

$$
|u(l, x, p)|^{2}=\omega^{2}(l, x) \sum_{i=1}^{m} H_{i}^{2}(l, z)=2 \omega(l, x) H_{n}(l, z),
$$


and define

$$
\lambda(x)=\max _{l \in \mathbf{S}^{1}} \lambda(l, x) .
$$

As a supremum of continuous functions, $x \mapsto \lambda(x)$ is only lower semi-continous and we must assume the existence of a continous upper bound in the subsequent result.

Proposition 3.4. Let $\sigma$ be a continuous function such that $\sigma^{2}$ dominates $\lambda$ in a neighbourhood of $\bar{x}$. Then

$$
\lim \sup _{\varepsilon \rightarrow 0+} \frac{\left\|u_{\varepsilon}\right\|_{\infty}}{\varepsilon} \leq\|\sigma \circ \bar{x} \cdot|\bar{p}|\|_{\infty} .
$$

Proof. On $\left[0, l_{f}\right]$, one has

$$
\begin{aligned}
\left|u_{\varepsilon}(l)\right|^{2} & =\varepsilon^{2}\left|u\left(l, \widetilde{z}_{\varepsilon}(\varepsilon l)\right)\right|^{2} \\
& \leq \varepsilon^{2} \sigma^{2} \circ \widetilde{x}_{\varepsilon}(\varepsilon l) \cdot\left|\widetilde{p}_{\varepsilon}(\varepsilon l)\right|^{2},
\end{aligned}
$$

so the result holds since the square root of the right hand side converges uniformly to $\sigma \circ \bar{x} \cdot|\bar{p}|$ as $\varepsilon$ goes to zero by continuity of $\sigma$.

3.3. Computations in Kepler case. Using the neat geometric coordinates of $\S 3.1$ on the three dimensional space of ellipses, we get

$$
\begin{gathered}
H_{n}(l, z)=\frac{\omega(l, x)}{2}\left(H_{1}^{2}+H_{2}^{2}\right)(l, z), \quad u(l, z)=\omega(l, x)\left(H_{1}, H_{2}\right)(l, z), \\
|u(l, z)|^{2}=\omega^{2}(l, x)\left(H_{1}^{2}+H_{2}^{2}\right)(l, z),
\end{gathered}
$$

with

$$
\begin{aligned}
F_{1}(l, x)= & \frac{P^{2}}{W^{2}}\left(\sin l \frac{\partial}{\partial e_{x}}-\cos l \frac{\partial}{\partial e_{y}}\right), \\
F_{2}(l, x)= & \frac{P^{2}}{W^{2}}\left(\frac{2 P}{W} \frac{\partial}{\partial P}\right. \\
& \left.+\left(\cos l+\frac{e_{x}+\cos l}{W}\right) \frac{\partial}{\partial e_{x}}+\left(\sin l+\frac{e_{y}+\sin l}{W}\right) \frac{\partial}{\partial e_{y}}\right),
\end{aligned}
$$

and

$$
\omega(l, x)=\frac{W^{2}}{P^{3 / 2}}, \quad W=1+e_{x} \cos l+e_{y} \sin l .
$$

Introducing mean motion, $n=a^{-3 / 2}$, and true anomaly, $\tau=l-\theta$, one gets

$$
\begin{aligned}
F_{1}(l, x)= & \frac{\left(1-e^{2}\right)^{2}}{n^{4 / 3} W^{2}}\left(-\frac{3 n e \sin \tau}{1-e^{2}} \frac{\partial}{\partial n}+\sin \tau \frac{\partial}{\partial e}-\cos \tau \frac{1}{e} \frac{\partial}{\partial \theta}\right) \\
F_{2}(l, x)= & \frac{\left(1-e^{2}\right)^{2}}{n^{4 / 3} W^{2}}\left(-\frac{3 n W}{1-e^{2}} \frac{\partial}{\partial n}+\left(\cos \tau+\frac{e+\cos \tau}{W}\right) \frac{\partial}{\partial e}\right. \\
& \left.+\left(\sin \tau+\frac{\sin \tau}{W}\right) \frac{1}{e} \frac{\partial}{\partial \theta}\right),
\end{aligned}
$$


with

$$
W=1+e \cos \tau
$$

As a result, the computation of Fourier series of $H_{n}$ (or $u$ ) are performed with respect to $\tau$ rather than $l$ so, as $\theta$ only appears through $\tau$,

$$
H_{n}(l, z)=\sum_{k \in \mathbf{Z}} c_{k}(n, e, p) e_{k}(l-\theta)=\sum_{k \in \mathbf{Z}} c_{k}(z) e_{k}(l)
$$

and coefficients verify

$$
c_{k}(z)=c_{k}(n, e, p) \bar{e}_{k}(\theta)
$$

Proposition 3.5. The adjoint $p_{\theta}$ is a linear first integral of $H$.

Proof. According to the previous remark, $\theta$ is cyclic in the averaged Hamiltonian.

The remarkable feature of the set $(n, e, \theta)$ of coordinates is the following $[7,18,19]$.

Proposition 3.6. The averaged Hamiltonian is Riemannian and orthogonal in $(n, e, \theta)$ coordinates,

$$
H(z)=\frac{1}{2 n^{5 / 3}}\left[9 n^{2} p_{n}^{2}+\frac{5}{2}\left(1-e^{2}\right) p_{e}^{2}+\frac{5-4 e^{2}}{2} \frac{p_{\theta}^{2}}{e^{2}}\right] .
$$

Fourier coefficients of the control are obviously obtained from (8-9), noting that

LEMMA 3.2. One has

$$
\frac{e+\cos \tau}{W}=-z-\frac{2 \sqrt{1-e^{2}}}{e} \sum_{k \geq 1} z^{k} \cos k \tau, \quad \frac{\sin \tau}{W}=-\frac{2}{e} \sum_{k \geq 1} z^{k} \sin k \tau,
$$

where $z=-e /\left(1+\sqrt{1-e^{2}}\right)$ is the only pole in the open unit disk of $W=1+e \cos \tau$.

We finally provide a continuous upper bound of the eigenvalues of the quadratic form associated with the control norm, allowing us to estimate precisely $\left\|u_{\varepsilon}\right\|_{\infty}$ as $\varepsilon \rightarrow 0+$.

Proposition 3.7. Eigenvalues of the quadratic form (7) are uniformly dominated by

$$
\sigma^{2}(n, e)=\frac{4\left(1-e^{2}\right)}{n^{2 / 3}}\left[\frac{(1+e)^{2}}{n^{4 / 3}}+1\right]+\frac{e}{n^{2 / 3}}\left[e+\sqrt{1-e^{2}}\right] .
$$

Proof. Since we have a rank two distribution (8-9) of vector fields (parameterized by $l \in \mathbf{S}^{1}$ ) on the three-dimensional manifold $X$, a simple computation shows that the maximum eigenvalue is

$$
\lambda(l, x)=\frac{\omega^{2}(l, x)}{2}\left[F_{1}^{2}+F_{2}^{2}+\sqrt{\left(F_{1}^{2}-F_{2}^{2}\right)^{2}+4\left(F_{1} \mid F_{2}\right)^{2}}\right](l, x),
$$

hence the result.

The resulting estimate provided by Proposition 3.4 depends only on the geodesic connecting the two prescribed points on the manifold. Complete quadrature for these geodesics are computed in the next section. 
3.4. Analysis of the averaged system. The main step in the analysis is to use further normalizations to obtain a geometric interpretation.

Proposition 3.8. In the elliptic domain, we set

$$
r=\frac{2}{5} n^{5 / 6}, \quad \varphi=\arcsin e,
$$

and the metric is isometric to

$$
g=\mathrm{d} r^{2}+\frac{r^{2}}{c^{2}}\left(\mathrm{~d} \varphi^{2}+G(\varphi) \mathrm{d} \theta^{2}\right)
$$

with

$$
c=\sqrt{2 / 5} \quad \text { and } \quad G(\varphi)=\frac{5 \sin ^{2} \varphi}{1+4 \cos ^{2} \varphi} .
$$

Geometric interpretation.. This normal form captures the main properties of the averaged orbital transfer. Indeed, we extract from $g$ two Riemannian metrics in dimension two

$$
g_{1}=\mathrm{d} r^{2}+r^{2} \mathrm{~d} \psi^{2}
$$

with $\psi=\varphi / c$ which is associated with orbital transfer where $\theta$ is kept fixed, and also

$$
g_{2}=\mathrm{d} \varphi^{2}+G(\varphi) \mathrm{d} \theta^{2}
$$

which represents the restriction to $r^{2}=c^{2}$.

3.4.1. Analysis of $\mathbf{g}_{\mathbf{1}}$. When $p_{\theta}$ vanishes, $\theta$ is constant. The corresponding extremals are geodesics of the Riemannian problem in dimension two defined by $\mathrm{d} \theta=$ 0 . We extend the elliptic domain to the meridian half-planes all isometric to

$$
\Sigma_{0}=\{n>0, e \in]-1,+1[\} .
$$

In polar coordinates $(r, \psi), \Sigma_{0}$ is defined by $\{r>0, \psi \in]-\pi /(2 c), \pi /(2 c)[\}$. This extension allows to go through the singularity corresponding to circular orbits. Geometrically, this describes transfers where the angle of the pericenter is kept fixed and $p_{\theta}=0$ corresponds to the transversality condition. Such a policy is clearly associated with steering the system towards circular orbits where the angle $\theta$ of the pericenter is not prescribed. An important physical subcase is the geostationary final orbit.

In the domain $\Sigma_{0}$, the metric $g_{1}=\mathrm{d} r^{2}+r^{2} \mathrm{~d} \psi^{2}$ is a polar metric isometric to the flat metric $\mathrm{d} x^{2}+\mathrm{d} z^{2}$ if we set $x=r \sin \psi$ and $z=r \cos \psi$.

We deduce the following proposition.

Proposition 3.9. The extremals of the averaged coplanar transfer in $\Sigma_{0}$ are straight lines in suitable coordinates, namely

$$
x=\frac{2^{3 / 2}}{5} n^{5 / 6} \sin \left(c^{-1} \arcsin e\right), \quad z=\frac{2^{3 / 2}}{5} n^{5 / 6} \cos \left(c^{-1} \arcsin e\right)
$$




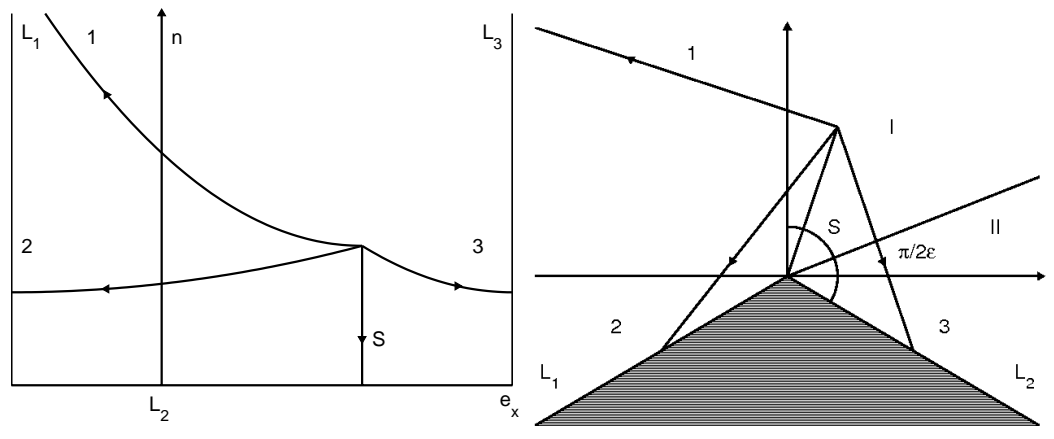

FIG. 1. Geodesics of the metric $g_{1}$ in $\left(n, e_{x}\right)$ and flat coordinates.

with $c=\sqrt{2 / 5}$. Since $c<1$, the domain is not convex and the metric $g_{1}$ is not complete.

Proof. The axis $e_{x}=0$ corresponds to circular orbits. Among the extremals, we have two types (see Fig. 1): complete curves of type 1, and non-complete curves of type 2 that meet the boundary of the domain. The domain is not geodesically convex and in subdomain II, the existence theorem fails. For each initial condition, there exists a separatrix $S$ which corresponds to a segment line in the orbital coordinates which is meeting $n=0$ in finite time. Its length gives the bound for a sphere to be compact.

In order to complete the analysis of $g$ and to understand the role of $g_{2}$, we present now the integration algorithm.

3.4.2. Integrability of the extremal flow. The integrability property is a consequence of the normal form only,

$$
g=\mathrm{d} r^{2}+r^{2}\left(\mathrm{~d} \varphi^{2}+G(\varphi) \mathrm{d} \theta^{2}\right)
$$

and the associated Hamiltonian is decomposed into

$$
H=\frac{1}{2} p_{r}^{2}+\frac{1}{r^{2}} H^{\prime}, \quad H^{\prime}=\frac{1}{2}\left(p_{\varphi}^{2}+\frac{p_{\theta}^{2}}{G(\varphi)}\right) .
$$

LEMma 3.3. The Hamiltonian vector field $\vec{H}$ admits three independent first integrals in involution, $H, H^{\prime}, p_{\theta}$, and is Liouville integrable.

To get a complete parameterization, we proceed as follows. We use the $(n, e, \theta)$ coordinates and write

$$
H=\frac{1}{4 n^{5 / 3}}\left[18 n^{2} p_{n}^{2}+H^{\prime \prime}\right]
$$

with

$$
H^{\prime \prime}=5\left(1-e^{2}\right) p_{e}^{2}+\frac{5-4 e^{2}}{e^{2}} p_{\theta}^{2}
$$


Lemma 3.4. Let $s=n^{5 / 3}$ then $s(t)$ is a polynomial of degree $2, s(t)=c_{1} t^{2}+$ $\dot{s}(0) t+s(0)$ with $s(0)=n^{5 / 3}(0), \dot{s}(0)=15 n(0) p_{n}(0)$ and $c_{1}=25 H / 2$.

Lemma 3.5. Let $\mathrm{d} T=\mathrm{d} t / 4 n^{5 / 3}$. If $H^{\prime \prime}(0) \neq 0$, then

$$
T(t)=\frac{1}{2 \sqrt{|\Delta|}}[\arctan L(s)]_{0}^{t}
$$

where $L(t)=(2 a t+b) / \sqrt{|\Delta|}, a=c_{1}, b=\dot{s}(0)$ and $\Delta=-25 H^{\prime \prime}(0) / 2$ is the discriminant of $s(t)$.

This allows to make the integration. Indeed if $H^{\prime \prime}=0, p_{e}=p_{\theta}=0$ and the trajectories are straight lines (the line $S$ in Fig. 1). Otherwise, we observe that $n^{5 / 3}(t)$ is known and depends only upon $n(0), p_{n}(0)$ and $H$ which can be fixed to $1 / 2$ by parameterizing by arc length. Hence, it is sufficient to integrate the flow associated with $H^{\prime \prime}$ using the parameter $\mathrm{d} T=\frac{\mathrm{d} t}{4 n^{5 / 3}}$ where $T$ is given by the previous lemma. Let $H^{\prime \prime}=c_{3}^{2}$ and $p_{\theta}=c_{2}$. Using $p_{e}=\dot{e} / 10\left(1-e^{2}\right)$, we obtain

$$
\dot{e}^{2}=\frac{20\left(1-e^{2}\right)}{e^{2}}\left[c_{3} e^{2}-\left(5-4 e^{2}\right) c_{2}^{2}\right]
$$

To integrate, we set $w=1-e^{2}$ for $\left.e \in\right] 0,1[$, so the equation takes the form

$$
\frac{\mathrm{d} w}{\mathrm{~d} T}=Q(w)
$$

where

$$
Q(w)=80 w\left[\left(c_{3}^{2}-c_{2}^{2}\right)-\left(c_{3}^{2}+4 c_{2}^{2}\right) w\right]
$$

with positive discriminant. Hence the solution is

$$
w=\frac{1}{2} \frac{c_{3}^{2}-c_{2}^{2}}{c_{3}^{2}+4 c_{2}^{2}}\left[1+\sin \left(\frac{4}{\sqrt{5}} \sqrt{c_{3}^{2}+4 c_{2}^{2}}\right) T+K\right],
$$

$K$ being a constant. We deduce that

$$
\theta(T)=\theta(0)+2 c_{2} \int_{0}^{T} \frac{1+4 w(s)}{1-w(s)} \mathrm{d} s
$$

where $\theta(0)$ can be set to 0 by symmetry. To conclude, we must integrate $(1+$ $4 w(s)) /(1-w(s))$ with $w=K_{1}(1+\sin x)$ and $x=(4 s / \sqrt{5}) \sqrt{c_{3}^{2}+4 c_{2}^{2}}+K$. Therefore, we must evaluate an integral of the form

$$
\int \frac{A+B \sin x}{C+D \sin x} \mathrm{~d} x
$$

More precisely, the formula is

$$
\int \frac{A+B \sin x}{C+D \sin x} \mathrm{~d} x=\frac{B}{D} x+A D-B C \int \frac{\mathrm{d} x}{C+D \sin x}
$$


with

$$
\int \frac{\mathrm{d} x}{C+D \sin x}=\frac{2}{\sqrt{C^{2}-D^{2}}} \arctan \left(\frac{C \tan (x / 2)+D}{\sqrt{C^{2}-D^{2}}}\right)
$$

and $C^{2}-D^{2}>0$. The previous lemmas and computations give

Proposition 3.10. For $H^{\prime \prime} \neq 0$, the solutions of $\vec{H}$ can be computed using elementary functions and

$$
\begin{aligned}
n(t) & =\left[\frac{25}{2} H t^{2}+15 n(0) p_{n}(0) t+n^{5 / 3}(0)\right]^{3 / 5} \\
e(t) & =\sqrt{1-K_{1}\left(1+\sin K_{2}(t)\right)} \\
\theta(t) & =\theta(0)+\frac{p_{\theta}}{2\left|p_{\theta}\right|} K_{3}\left[-4 x+\frac{10}{K_{3}} \arctan \frac{\left(1-K_{1}\right) \tan (x / 2)-K_{1}}{K_{3}}\right]_{K}^{K_{2}(t)}
\end{aligned}
$$

with

$$
\begin{gathered}
K=\arcsin \left(\frac{1-e(0)^{2}}{K_{1}}-1\right), \quad K_{1}=\frac{1}{2} \frac{H^{\prime \prime}(0)-p_{\theta}^{2}}{H^{\prime \prime}(0)+4 p_{\theta}^{2}}, \\
K_{2}(t)=\frac{4}{\sqrt{5}}\left(T(t) \sqrt{H^{\prime \prime}(0)+4 p_{\theta}^{2}}+K\right), \quad K_{3}=\sqrt{\frac{5 p_{\theta}^{2}}{H^{\prime \prime}(0)+4 p_{\theta}^{2}}} .
\end{gathered}
$$

For $H^{\prime \prime}=0$, they are straight lines.

REMARK 3.3. The above formulas give the complete solution of the associated Hamilton-Jacobi Equation.

3.4.3. Geometric properties of $\mathbf{g}_{2}$. The previous integration algorithm shows that the extremals of this metric describe the evolution of the angular variables $\theta$ and $\varphi$ parameterized by $\mathrm{d} T=\mathrm{d} t / r(t)^{2}$ where $r(t)^{2}$ is a second order polynomial whose coefficients depend only upon the energy level $H$ fixed to $1 / 2, r(0)$ and $p_{r}(0)$. We now give some basic properties of $g_{2}$.

LEMMA 3.6. The metric $g_{2}$ can be extended to an analytic metric on the whole sphere $\mathbf{S}^{2}$, where $\theta$ and $\varphi$ are spherical coordinates with two polar singularities at $\varphi=0$ or $\pi$ corresponding to $e=0$, whereas the equator corresponds to $e=1 ; \theta$ is an angle of revolution. The meridians are projections on $\mathbf{S}^{2}$ of the extremals of $g_{1}$.

LEMMA 3.7. The two transformations $(\varphi, \theta) \mapsto(\varphi,-\theta)$ and $(\varphi, \theta) \mapsto(\pi-\varphi, \theta)$ are isometry of $g_{2}$. This induces the following symmetries for the extremal flow:

- if $p_{\theta} \mapsto-p_{\theta}$ then we have two extremals of same length symmetric with respect to the meridian $\theta=0$,

- if $p_{\varphi} \mapsto-p_{\varphi}$ then we have two extremals of same length intersecting on the antipodal parallel, $\varphi=\pi-\varphi(0)$.

Such properties (illustrated on Fig. 3.4.3) are shared by the following one-parameter family of metrics. 

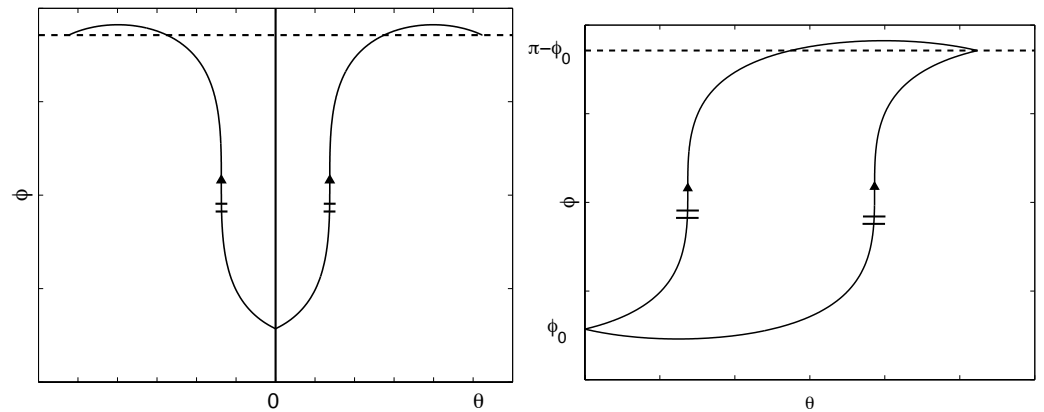

FIG. 2. Action of the symmetry group on the extremals.

Metrics induced by the flat metric on oblate ellipsoid of revolution.. We consider the flat metric of $\mathbf{R}^{3}, g=\mathrm{d} x^{2}+\mathrm{d} y^{2}+\mathrm{d} z^{2}$, restricted to the ellipsoid defined by

$$
x=\sin \varphi \cos \theta, y=\sin \varphi \sin \theta, z=\mu \cos \varphi
$$

where $\mu \in] 0,1[$. A simple computation leads to

$$
E_{\mu}(\varphi) \mathrm{d} \varphi^{2}+\sin ^{2} \varphi \mathrm{d} \theta^{2}
$$

for the restricted metric, where $E_{\mu}(\varphi)=\mu^{2}+\left(1-\mu^{2}\right) \cos ^{2} \varphi$, and we can write

$$
g_{2}=\frac{1}{E_{\mu}(\varphi)}\left(E_{\mu}(\varphi) \mathrm{d} \varphi^{2}+\sin ^{2} \varphi \mathrm{d} \theta^{2}\right)
$$

where $\mu=1 / \sqrt{5}$. We deduce the following lemma.

LEMMA 3.8. The metric $g_{2}$ is conformal to the flat metric restricted to an oblate ellipsoid of revolution with parameter $\mu=1 / \sqrt{5}$.

3.4.4. A global optimality result with application to orbital transfer. In this section, we consider an analytic metric on $\mathbf{R}_{+} \times \mathbf{S}^{2}$

$$
g=\mathrm{d} r^{2}+\left(\mathrm{d} \varphi^{2}+G(\varphi) \mathrm{d} \theta^{2}\right)
$$

and let $H$ be the associated Hamiltonian. We fix the parameterization to arc length by restricting to the level set $H=1 / 2$. Let $x_{1}, x_{2}$ be two extremal curves starting from the same initial point $x_{0}$ and intersecting at some positive $\bar{t}$. We get the relations

$$
r_{1}(\bar{t})=r_{2}(\bar{t}), \quad \varphi_{1}(\bar{t})=\varphi_{2}(\bar{t}), \quad \theta_{1}(\bar{t})=\theta_{2}(\bar{t}),
$$

and from Lemma 3.4, we deduce that

Lemma 3.9. Both extremals $x_{1}$ and $x_{2}$ share the same $p_{r}(0)$ and for each $t$, $r_{1}(t)=r_{2}(t)$.

If we consider now the integral curves of $H^{\prime}$ where $H=(1 / 2) p_{r}^{2}+H^{\prime} / r^{2}$ on the fixed induced level and parameterize these curves using $\mathrm{d} T=\mathrm{d} t / r^{2}$, we deduce the following characterization. 
Proposition 3.11. The following conditions are necessary and sufficient to characterize extremals of $H^{\prime} \neq 0$ intersecting with same length

$$
\varphi_{1}(\bar{T})=\varphi_{2}(\bar{T}) \quad \text { and } \quad \theta_{1}(\bar{T})=\theta_{2}(\bar{T})
$$

together with the compatibility condition

$$
\bar{T}=\int_{0}^{\bar{t}} \frac{\mathrm{d} t}{r^{2}(t)}=\left[\frac{2}{\sqrt{\Delta}} \arctan L(t)\right]_{t=0}^{\bar{t}} .
$$

TheOREM 3.2. A necessary global optimality condition for an analytic metric on $\mathbf{R}_{+} \times \mathbf{S}^{1}$ normalized to

$$
g=\mathrm{d} r^{2}+r^{2}\left(\mathrm{~d} \varphi^{2}+G(\varphi) \mathrm{d} \theta^{2}\right)
$$

is that the injectivity radius be greater than or equal to $\pi$ on the sphere $r=1$, the bound being reached by the flat metric in spherical coordinates.

Proof. We observe that in the flat case, the compatibility condition cannot be satisfied. Moreover, the injectivity radius on $\mathbf{S}^{2}$ is $\pi$ corresponding to the half-length of a great circle. For the analytic metric on $\mathbf{S}^{2}$ under consideration, the injectivity radius is the length of the conjugate point at minimum distance or the half-length of a closed geodesic [17]. The conjugate point is, in addition, a limit point of the separating line. Hence, if the injectivity radius is smaller than $\pi$, we have two minimizers for the restriction of the metric on $\mathbf{S}^{2}$ which intersects with a length smaller than $\pi$. We shall show that it corresponds to a projection of two extremals $x_{1}$ and $x_{2}$ which intersect with same length.

For such extremals $r(0)=1$, we set $p_{r}(0)=\varepsilon, H=1 / 2$ and we get

$$
2 H^{\prime}=p_{\varphi}^{2}(0)+\frac{p_{\theta}^{2}(0)}{G(\varphi(0))}=\lambda^{2}(\varepsilon), \quad \lambda(\varepsilon)=\sqrt{1-\varepsilon^{2}} .
$$

If $t_{1}$ is the injectivity radius on the level set $H^{\prime}=1 / 2$, for $H^{\prime}=\lambda^{2}(\varepsilon) / 2$ and $p_{r}(0)=\varepsilon$, it is rescaled to $T_{1}=t_{1} / \lambda(\varepsilon)$. The compatibility relation for $\bar{T}=T_{1}$ then gives

$$
T_{1}=\arctan \frac{\bar{t}+\varepsilon}{\lambda(\varepsilon)}-\arctan \frac{\varepsilon}{\lambda(\varepsilon)}
$$

Clearly, the maximum of the right member is $\pi$, taking $\varepsilon<0,|\varepsilon| \rightarrow 1$. Hence, it can be satisfied since $t_{1}<\pi$. The flat case shows that it is the sharpest bound. $\quad \square$ By homogeneity, we deduce the following corollary.

Corollary 3.1. If the metric is normalized to $\mathrm{d} r^{2}+\left(r^{2} / c^{2}\right)\left(\mathrm{d} \varphi^{2}+G(\varphi) \mathrm{d} \theta^{2}\right)$, then the bound for the injectivity radius on $r^{2}=c^{2}$ is $c \pi$.

3.4.5. Riemannian curvature and injectivity radius in orbital transfer. Using standard formulæ from Riemannian geometry [17], we have the following proposition. 
Proposition 3.12. Let $g$ be a smooth metric of the form $\mathrm{d} r^{2}+r^{2}\left(\mathrm{~d} \varphi^{2}+G(\varphi) \mathrm{d} \theta^{2}\right)$. The only non-zero component of the Riemann tensor is

$$
R_{2323}=r^{2}\left[-\frac{G^{\prime \prime}(\varphi)}{2}-G(\varphi)+\frac{G^{\prime}(\varphi)^{2}}{4 G(\varphi)}\right]
$$

which takes the form $R_{2323}=-r^{2} F\left(F^{\prime \prime}+F\right)$ if we set $G(\varphi)=F^{2}(\varphi)$. We have therefore $R_{2323}=0$ if and only if $F(\varphi)=A \sin \left(\varphi+\varphi_{0}\right)$ which is induced by the flat case in spherical coordinates.

Hence, the main non-zero sectional curvature of the metric is

$$
K=\frac{R_{2323}}{\left|\frac{\partial}{\partial \theta} \wedge \frac{\partial}{\partial \varphi}\right|^{2}}
$$

and computing this term in the case of orbital transfer, we get:

Lemma 3.10. The sectional curvature in the plane $(\varphi, \theta)$ is given by

$$
K=\frac{\left(1-24 \cos ^{2} \varphi-16 \cos ^{4} \varphi\right)}{r^{2}\left(1+4 \cos ^{2} \varphi\right)^{2}}
$$

and $K \rightarrow 0$ as $r \rightarrow+\infty$.

Proposition 3.13. The Gauss curvature of the metric of $g_{2}=\mathrm{d} \varphi^{2}+G(\varphi) \mathrm{d} \theta^{2}$ with $G(\varphi)=\left(\sin ^{2} \varphi\right) /\left(1+4 \cos ^{2} \varphi\right)$ is

$$
K=\frac{5\left(1-8 \cos ^{2} \varphi\right)}{\left(1+4 \cos ^{2} \varphi\right)^{2}} .
$$

Theorem 3.3. The Gauss curvature of $g_{2}$ is negative near the poles and maximum at the equator. The injectivity radius is $\pi / \sqrt{5}$ and is reached by the shortest conjugate point along the equator.

Proof. Clearly $K$ is maximum and equal to five along the equator which is an extremal solution. Hence a direct computation gives that the shortest conjugate point is on the equator with length $\pi / \sqrt{5}$. It corresponds to the injectivity radius if the halflength of a shortest periodic extremal is greater than $\pi / \sqrt{5}$. Simple closed extremals are computed in [8] using the integrability property and a simple reasoning gives that the shortest corresponds to meridians whose length is $2 \pi$. Hence the result is proved.

Corollary 3.2. Since $\pi / \sqrt{5}<\pi \sqrt{2 / 5}$, the necessary optimality condition of Theorem 3.3 is not satisfied in orbital transfer for the extension of the metric to $\mathbf{R}^{+} \times \mathbf{S}^{2}$.

\subsubsection{Cut locus on $\mathrm{S}^{2}$ and global optimality results in orbital transfer.}

From the previous section, the computation of the injectivity radius for the metric on $\mathbf{S}^{2}$ is not sufficient to conclude about global optimality. A more complete analysis is necessary to evaluate the cut locus. This analysis requires numerical simulations. The main results are $[8,11]$ : 


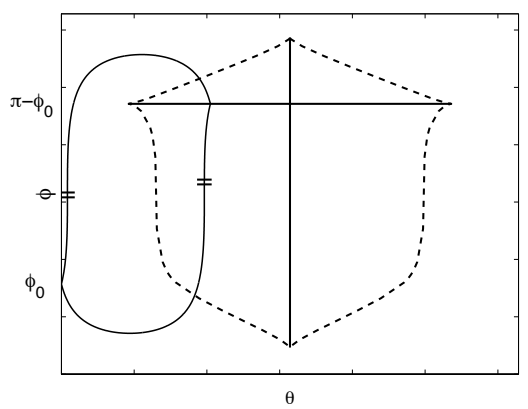

FIG. 3. Conjugate and cut loci in averaged orbital transfer.

Proposition 3.14. For the metric $g_{2}$ on $\mathbf{S}^{2}$, they are exactly five simple closed geodesics modulo rotations around the poles, the shortest being meridians with length $2 \pi$, the longest the equator with length $2 \pi \sqrt{5}$.

THEOREM 3.4. Except for poles, the conjugate locus is a deformation of a standard astroid with axial symmetry and two cusps located on the antipodal parallel. With the exception of poles, the cut locus is a simple segment, located on the antipodal parallel, with axial symmetry, and whose extremities are cusps points of the conjugate locus. For a pole, the cut locus is reduced to the antipodal pole.

Proof. The proof is made by direct analysis of the extremal curves. The main problem is to prove that the separating line is given by points on the antipodal parallel where, because of the isometry $\varphi \rightarrow \pi-\varphi$, two extremals curves with same length intersect. This property cannot occur before. The results are represented Fig. 3.

Geometric interpretation and comments.. The metric is conformal to the restriction of the flat metric to an oblate ellipsoid of revolution. For such a metric, the cut locus is known since Jacobi and is similar to the one represented on Fig. 3. It is a remarkable property that there is no bifurcation of the cut locus when the metric is deformed by the factor $E_{\mu}(\varphi)$ In orbital transfer for instance, the Gauss curvature is not positive. On $\mathbf{S}^{2}$, relations between the conjugate and cut loci allow to deduce the cut locus from the conjugate locus. ${ }^{2}$ The conjugate locus can also easily be computed using the code [12]. It can also be deduced by inspecting the extremal flow only, the conjugate locus being an envelope.

Finally, we observe that in order to have intersecting minimizers, we must cross the equator $\varphi=\pi$, that is $e=1$. The same is true for conjugate points. Hence we deduce

TheOREM 3.5. Conjugate loci and separating lines of the averaged Kepler metric are always empty in the spaces of ellipses where $e \in[0,1[$.

\footnotetext{
${ }^{2}$ For instance, a domain bounded by two intersecting minimizing curves must contain a conjugate point.
} 
3.5. The averaged system in the tangential case. An interesting question is to analyze if the averaged system in the tangential case where the control is oriented along $F_{t}$ retains similar properties [10]. The first step is to compute the corresponding averaged system.

Proposition 3.15. If the control is oriented along $F_{t}$ only, the averaged Hamiltonian associated with energy minimization is

$$
H_{t}=\frac{1}{2 n^{5 / 3}}\left[9 n^{2} p_{n}^{2}+\frac{4\left(1-e^{2}\right)^{3 / 2}}{1+\sqrt{1-e^{2}}} p_{e}^{2}+\frac{4\left(1-e^{2}\right)}{1+\sqrt{1-e^{2}}} \frac{p_{\theta}^{2}}{e^{2}}\right]
$$

and corresponds to the Riemannian metric

$$
g_{t}=\frac{\mathrm{d} n^{2}}{9 n^{1 / 3}}+\frac{n^{5 / 3}}{4}\left[\frac{1+\sqrt{1-e^{2}}}{\left(1-e^{2}\right)^{3 / 2}} \mathrm{~d} e^{2}+\frac{1+\sqrt{1-e^{2}}}{\left(1-e^{2}\right)} e^{2} \mathrm{~d} \theta^{2}\right]
$$

where $(n, e, \theta)$ remain orthogonal coordinates.

3.5.1. Construction of the normal form. We proceed as in Section 3.4 and set

$$
r=\frac{2}{5} n^{5 / 6}, \quad e=\sin \varphi \sqrt{1+\cos ^{2} \varphi}
$$

The metric becomes

$$
g_{t}=\mathrm{d} r^{2}+\frac{r^{2}}{c^{2}}\left(\mathrm{~d} \varphi^{2}+G(\varphi) \mathrm{d} \theta^{2}\right), \quad c=\frac{2}{5}<1,
$$

and

$$
G(\varphi)=\sin ^{2} \varphi\left(\frac{1-(1 / 2) \sin ^{2} \varphi}{1-\sin ^{2} \varphi}\right)^{2}
$$

Hence the normal form is similar to the full control case. As before, we introduce the metrics

$$
g_{1}=\mathrm{d} r^{2}+r^{2} \mathrm{~d} \psi^{2}, \quad \psi=\varphi / c
$$

and

$$
g_{2}=\mathrm{d} \varphi^{2}+G(\varphi) \mathrm{d} \theta^{2}
$$

The main difference with the full control case will concern the singularities of the function $G$.

3.5.2. Metrics $\mathrm{g}_{1}$ and $\mathrm{g}_{2}$. The metric $g_{1}$ corresponds again to transfer to circular orbits and is the polar form of the flat metric $\mathrm{d} x^{2}+\mathrm{d} z^{2}$, if $x=r \sin \psi$ and $z=r \cos \psi$.

The normal form reveals the same homogeneity property between the full control and the tangential case, so the metric $g_{2}$ can be used to make a similar optimality analysis, evaluating the conjugate and cut locus. But the metric $g_{2}$ cannot be 
interpreted as a smooth metric on $\mathbf{S}^{2}$. This can be seen by computing the Gauss curvature.

Proposition 3.16. The Gauss curvature of $g_{2}$ is given by

$$
K=\frac{\left(3+\cos ^{2} \varphi\right)\left(\cos ^{2} \varphi-2\right)}{\left(1+\cos ^{2} \varphi\right) \cos ^{2} \varphi}
$$

In particular $K \rightarrow-\infty$ when $\varphi \rightarrow \pi / 2$, and since $K<0$, the conjugate locus of any point is empty.

Nevertheless, the extremals can be smoothly extended through the singular boundary of the domain, the equator $\varphi=\pi / 2$.

3.5.3. Integration of the extremal flow. The algorithm based on the normal form is similar to the bi-input case, but we compare the respective transcendence. The Hamiltonian is written

$$
H=\frac{1}{4 n^{5 / 3}}\left[18 n^{2} p_{n}^{2}+H^{\prime \prime}\right]
$$

where $H^{\prime \prime}$ now takes the form

$$
H^{\prime \prime}=\frac{8\left(1-e^{2}\right)^{3 / 2}}{1+\sqrt{1-e^{2}}} p_{e}^{2}+\frac{8\left(1-e^{2}\right)}{1+\sqrt{1-e^{2}}} \frac{p_{\theta}^{2}}{e^{2}} .
$$

We set $H^{\prime \prime}=c_{3}^{2}, p_{\theta}=c_{2}$, and from

$$
p_{e}=4 n^{5 / 3} \frac{\left(1+\sqrt{1-e^{2}}\right) e}{16\left(1-e^{2}\right)^{3 / 2}}
$$

we obtain

$$
\left(\frac{\mathrm{d} w}{\mathrm{~d} T}\right)^{2}=\frac{Q(w)}{(1+w)^{2}}
$$

where $w=\sqrt{1-e^{2}}, T$ is as in the bi-input case, and $Q$ is the fourth-order polynomial

$$
Q(w)=32 w\left[c_{3}^{2}\left(1-w^{2}\right)(1+w)-8 c_{2}^{2} w^{2}\right] .
$$

Hence, the integration requires the computation of the elliptic integral

$$
\int \frac{\mathrm{d} w(1+w)}{\sqrt{Q(w)}}
$$

which has an additional complexity. It is related to the pole of order 2 of the metric at the equator. See $[9,15]$ for both aspects.

3.5.4. Conclusion in both cases. The previous analysis shows that the full control case and the tangential one admit a uniform representation in coordinates $(\theta, \varphi)$. In particular, it allows to make a continuation between the respective Hamiltonians, i.e., between the respective functions $G$. A correction has to be made between orbit elements $e$ which are respectively defined by

$$
e=\sin \varphi \quad \text { versus } \quad e=\sin \varphi \sqrt{1+\cos ^{2} \varphi}
$$



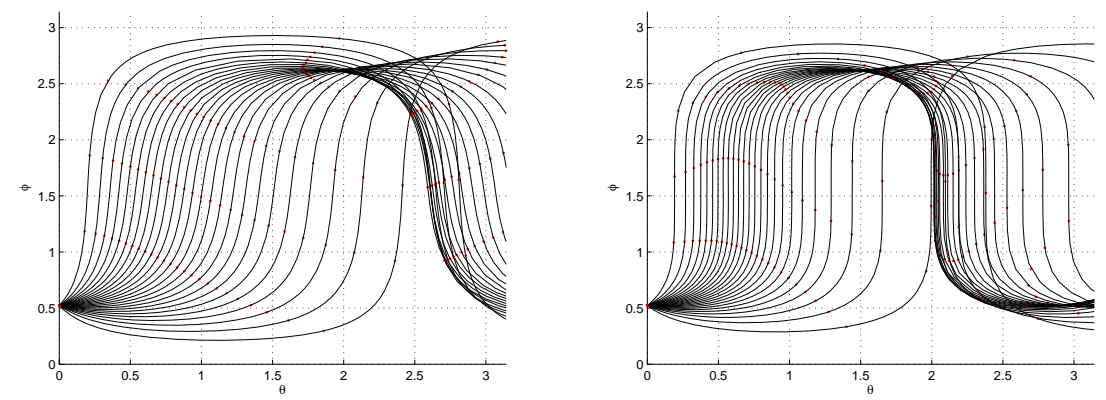

FIG. 4. Extremal flow of $g_{2}$ in the full control and tangential cases, in the $(\theta, \varphi)$-coordinates, starting from $\varphi=\pi / 6$.

The flows in the two cases are presented on Fig. 4 and reveal the similar structure. The conjugate locus is reached after having crossed the equator. On Fig. 5 and 6 we present a first continuation result, in the tangential case, showing the convergence of the method from the averaged to the non averaged trajectory for specific transfer orbits.

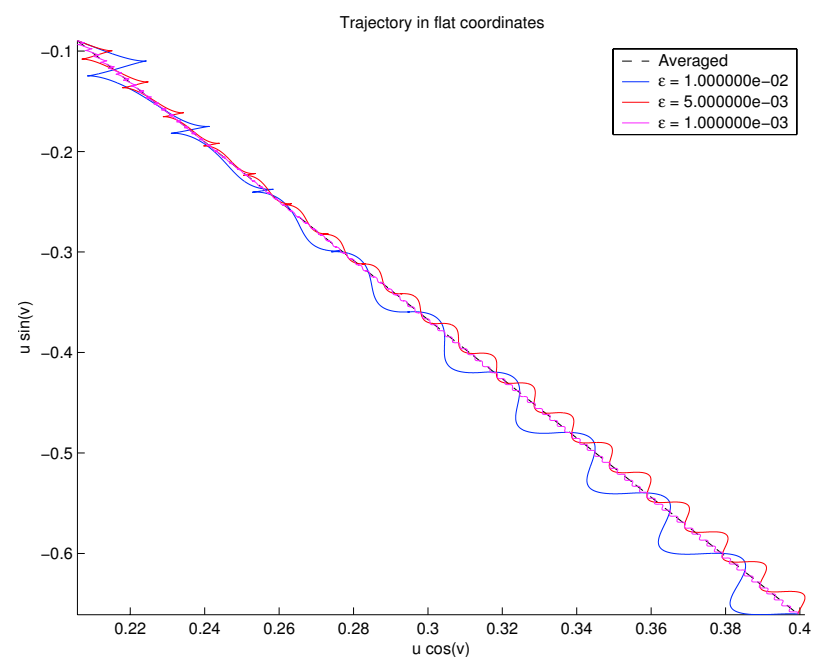

FIG. 5. Computation by continuation of the non-averaged solution. The averaged trajectories are clearly nice approximations of the optimal ones of the original system. Hence, convergence of the underlying shooting method to compute the non-averaged minimizing trajectory is easily obtained.

3.6. Conjugate and cut loci on a two-sphere of revolution. The problem of computing the conjugate and cut loci in orbital transfer is connected to a very old geometric problem which goes back to Jacobi and is briefly introduced next.

Definition 3.1. The two-sphere $\mathbf{S}^{2}$ endowed with a smooth metric of the form $\mathrm{d} \varphi^{2}+G(\varphi) \mathrm{d} \theta^{2}$ in spherical coordinates is called two-sphere of revolution.

Many of them can be realized as Riemannian surfaces of revolution embedded 


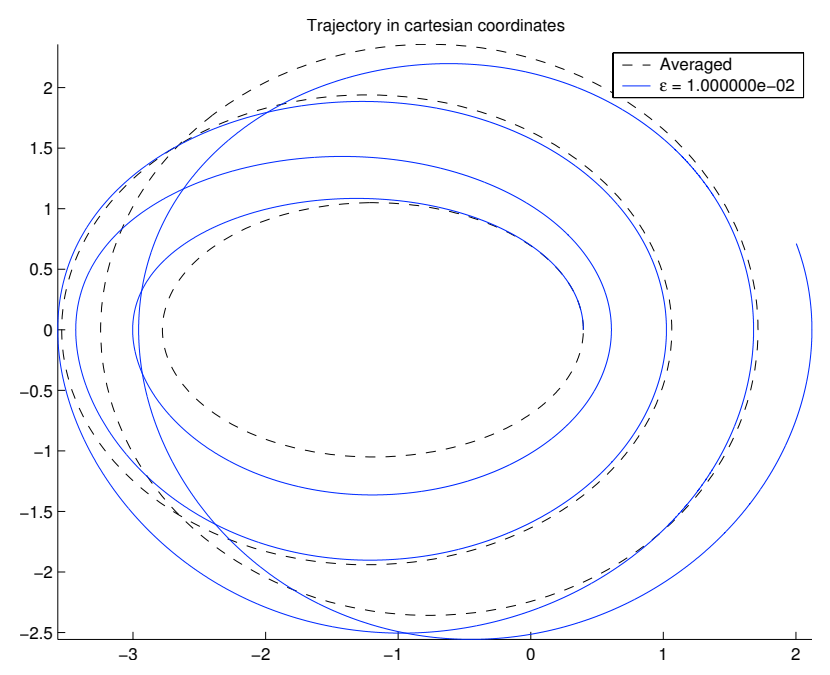

FIG. 6. Trajectory of a non-averaged solution for $\varepsilon=1 e-2$ with $(e(0), n(0))=(7.5 e-1,5 e-1)$ and $\left(e\left(t_{f}\right), n\left(t_{f}\right)\right)=(5 e-2,3 e-1)$. Dashed ellipses are averaged ellipses and provide a good approximation of the motion.

in $\mathbf{R}^{3}$ by rotating on a smooth curve homeomorphic to a half-circle. The classical examples are the following:

- Round sphere $\mathbf{S}^{2}$. It is constructed restricting the Euclidian metric to $\mathbf{S}^{2}$ and given by $\mathrm{d} \varphi^{2}+\sin ^{2}(\varphi) \mathrm{d} \theta^{2}$.

- Oblate ellipsoid of revolution $O(\mu)$. If we restrict the Euclidian metric to the surface

$$
x=\sin \varphi \cos \theta, \quad y=\sin \varphi \sin \theta, \quad z=\mu \cos \varphi,
$$

with $\mu<1$, the metric is $\left(1-\left(1-\mu^{2}\right) \sin ^{2} \varphi\right) \mathrm{d} \varphi^{2}+\sin ^{2}(\varphi) \mathrm{d} \theta^{2}$ which can be set in the form $\mathrm{d} \varphi^{2}+G(\varphi) \mathrm{d} \theta^{2}$ using a quadrature.

We recall some basic properties on the ellipsoid of revolution.

Proposition 3.17. On an oblate ellipsoid of revolution,

- the Gauss curvature is monotone increasing from the North pole to the equator,

- the cut locus of a point which is not a pole is a subarc of the antipodal parallel,

- the conjugate locus of a point which is not a pole has a standard astroid shape with four cusps.

The simple structure of the cut locus is a consequence of [31].

TheOREM 3.6. Let $\mathrm{d} \varphi^{2}+G(\varphi) \mathrm{d} \theta^{2}$ be a metric on a two-sphere of revolution. We assume:

- The transformation $\varphi \longrightarrow \pi-\varphi$ is an isometry i.e. $G(\pi-\varphi)=G(\varphi)$,

- The Gauss curvature $K$ is monotone non decreasing along a meridian from the North pole to the equator. 
Then, the cut locus of a point not a pole is a simple branch located on the antipodal parallel.

Application. Let $g_{\lambda}$ be the family of analytic metrics on $\mathbf{S}^{2}$ defined by

$$
g_{\lambda}=\mathrm{d} \varphi^{2}+G_{\lambda}(\varphi) \mathrm{d} \theta^{2}, \quad G_{\lambda}(\varphi)=\frac{(1+\lambda)^{2} \sin ^{2} \varphi}{\left(1+\lambda \cos ^{2} \varphi\right)}, \quad \lambda \geq 0
$$

The Gauss curvature is given by

$$
K_{\lambda}=-\frac{1}{\sqrt{G}} \frac{\partial^{2} \sqrt{G}}{\partial^{2} \varphi}=\frac{(1+\lambda)\left(1-2 \lambda \cos ^{2} \varphi\right)}{\left(1+\lambda \cos ^{2} \varphi\right)^{2}} .
$$

Hence if $0<\lambda \leq 2$, then $K_{\lambda}$ is monotone non decreasing from the North pole to the equator and the previous theorem asserts that the one parameter family has a cut locus reduced to a simple branch for $\lambda \in] 0,2]$.

If $\lambda>2$ the Gaussian curvature $K_{\lambda}$ is not monotone and the result cannot be applied. In particular the orbit transfer with full control corresponds to $\lambda=4$, while at the limit case $\lambda=+\infty$ a singularity appears. The Riemannian metric has a pole at the equator, the situation being similar to the one occuring when the thrust is only tangential. Hence Theorem 3.6 has to be refined to deal with such situations. The final result is coming from [9].

We consider a metric of the form $g=\varphi^{2}+G(\varphi) \mathrm{d} \theta^{2}$ where $G^{\prime}$ is non zero on $] 0, \frac{\pi}{2}[$ and $G(\pi-\varphi)=G(\varphi)$.

DEFINITION 3.2. The first return mapping to the equator is the map

$$
\left.\Delta \theta: p_{\theta} \in\right] 0, \sqrt{G}(\pi / 2)\left[\mapsto \Delta \theta\left(p_{\theta}\right)\right.
$$

that is the $\theta$-variation of the extremal parameterized by arc length and associated with the adjoint vector component $p_{\theta}$. The extremal flow is called tame if the first return mapping is monotone non-increasing for $\left.p_{\theta} \in\right] 0, \sqrt{G}(\pi / 2)[$.

THEOREM 3.7. In the tame case, the cut locus of a point different from a pole is a subset of the antipodal parallel. If moreover $\Delta \theta^{\prime}\left(p_{\theta}\right)<0<\Delta \theta^{\prime \prime}\left(p_{\theta}\right)$ on $] 0, \sqrt{G}(\pi / 2)[$, then the conjugate locus of such a point has exactly four cusps.

REMARK 3.4. This result can be extended to the singular case where the metric has poles at the equator.

\section{Energy minimization in the Earth-Moon space mission with low} thrust.

4.1. The N-body problem. In this section, we follow mainly [25] (see also [27] and [32]). Consider $N$ point masses $m_{1}, \ldots, m_{N}$ moving in a Galilean reference system $\mathbf{R}^{3}$ where the only forces acting are their mutual attractions. If $q=\left(q_{1}, \ldots, q_{N}\right) \in$ $\mathbf{R}^{3 N}$ is the state and $p=\left(p_{1}, \ldots, p_{N}\right)$ is the momentum vector, the equations of 
motion are

$$
\dot{q}=\frac{\partial H}{\partial p}, \quad \dot{p}=-\frac{\partial H}{\partial q}
$$

where the Hamiltonian is

$$
H=\sum_{i=1}^{N} \frac{\left|p_{i}\right|^{2}}{2 m_{i}}-U, \quad U(q)=\sum_{1 \leq i<j \leq N}^{N} \frac{G m_{i} m_{j}}{\left|q_{i}-q_{j}\right|}
$$

A subcase is the coplanar situation where the $N$ masses are in a plane $\mathbf{R}^{2}$. In this case the Galilean reference frame can be replaced by a rotating frame defined by

$$
K=\left[\begin{array}{cc}
0 & 1 \\
-1 & 0
\end{array}\right], \quad \exp (\omega t K)=\left[\begin{array}{cc}
\cos \omega t & \sin \omega t \\
-\sin \omega t & \cos \omega t
\end{array}\right]
$$

and introducing a set of coordinates which uniformly rotates with frequency $\omega$, one defines the symplectic transformation

$$
u_{i}=\exp (\omega t K) q_{i}, \quad v_{i}=\exp (\omega t K)
$$

A standard computation gives the Hamiltonian of the $N$-body problem in rotating coordinates,

$$
H=\sum_{i=1}^{N} \frac{|v|^{2}}{2 m_{i}}-\sum_{i=1}^{N} w^{t} u_{i} K v_{i}-\sum_{1 \leq i<j \leq N}^{N} \frac{G m_{i} m_{j}}{\left|q_{i}-q_{j}\right|}
$$

In particular, the Kepler problem in rotating coordinates up to a normalization has the following Hamiltonian

$$
H=\frac{|p|^{2}}{2}-{ }^{t} q K p-\frac{1}{|q|}
$$

4.2. The circular restricted 3 -body problem in Jacobi coordinates. The following representation of the Earth-Moon problem fits in the so-called circular restricted 3-body problem. In the rotating frame, the Earth which is the biggest primary planet with mass $1-\mu$ is located at $(-\mu, 0)$ while the Moon with mass $\mu$ is located at $(1-\mu, 0)$ (the small parameter being $\mu \simeq 1.2153 e-2)$. We note $z=x+i y$ the position of the spacecraft, and $\rho_{1}, \rho_{2}$ the distances to the primaries,

$$
\begin{aligned}
& \rho_{1}=\sqrt{(x+\mu)^{2}+y^{2}}, \\
& \rho_{2}=\sqrt{(x-1+\mu)^{2}+y^{2}} .
\end{aligned}
$$

The equation of motion is

$$
\ddot{z}+2 i \dot{z}-z=-(1-\mu) \frac{z+\mu}{\rho_{1}^{3}}-\mu \frac{z-1+\mu}{\rho_{2}^{3}}
$$


that is

$$
\ddot{x}-2 \dot{y}-x=\frac{\partial V}{\partial x}, \quad \ddot{y}+2 \dot{x}-y=\frac{\partial V}{\partial y},
$$

where $-V$ is the potential of the system defined by

$$
V=\frac{1-\mu}{\rho_{1}^{3}}+\frac{\mu}{\rho_{2}^{3}} .
$$

The system can be written using Hamiltonian formalism setting

$$
q_{1}=x, \quad q_{2}=y, \quad p_{1}=\dot{x}-y, \quad q_{2}=\dot{y}+x,
$$

and the Hamiltonian describing the motion writes

$$
H_{0}\left(q_{1}, q_{2}, p_{1}, p_{2}\right)=\frac{1}{2}\left(p_{1}^{2}+p_{2}^{2}\right)+p_{1} q_{2}-p_{2} q_{1}-\frac{1-\mu}{\rho_{1}}-\frac{\mu}{\rho_{2}} .
$$

4.3. Jacobi integral, Hill regions and equilibrium points. The Jacobi integral using Hamiltonian formalism is simply the Hamiltonian $H_{0}$ which gives

$$
H_{0}(x, y, \dot{x}-y, \dot{y}+x)=\frac{\dot{x}^{2}+\dot{y}^{2}}{2}-\Omega(x, y)
$$

where

$$
\Omega(x, y)=\frac{1}{2}\left(x^{2}+y^{2}\right)+\frac{1-\mu}{\rho_{1}}+\frac{\mu}{\rho_{2}} .
$$

Solutions are confined on the level set

$$
\frac{\dot{x}^{2}+\dot{y}^{2}}{2}-\Omega(x, y)=h, \quad h \text { constant. }
$$

The Hill domain for the value $h$ is the region where the motion can occur, that is $\left\{(x, y) \in \mathbf{R}^{2} \mid \Omega(x, y)+h \geq 0\right\}$.

The equilibrium points of the problem are well known. They split into two different types:

- Euler points. They are the collinear points denoted $L_{1}, L_{2}$ and $L_{3}$ located on the line $y=0$ defined by the primaries. For the Earth-Moon problem they are given by

$$
x_{1} \simeq-1.0051, \quad x_{2} \simeq 8.369 e-1, \quad x_{3} \simeq 1.1557 .
$$

- Lagrange points. The two points $L_{4}$ and $L_{5}$ form with the two primaries an equilateral triangle.

Some important information about stability of the equilibrium points are provided by the eigenvalues of the linearized system. The linearized matrix evaluated at points $L_{1}$, $L_{2}$ or $L_{3}$ admits two real eigenvalues, one being strictly positive, and two imaginary ones. The collinear points are consequently not stable. In particular, the eigenvalues 
of the linearized matrix evaluated at $L_{2}$ with $\mu=1.2153 e-2$ are approximately \pm 2.931837 and $\pm 2.334248 i$. When it is evaluated at $L_{4}$ or $L_{5}$, the linearized matrix has two imaginary eigenvalues since $\mu<\mu_{1}=(1 / 2)(1-\sqrt{69} / 9)$ in the Earth-Moon system. Points $L_{4}$ and $L_{5}$ are thus stable according to Arnold stability theorem [25]. See also [24] for a review of mission design techniques using the equilibrium points.

4.4. The continuation method. The mathematical continuation method in the restricted circular problem is omnipresent in Poincaré's work, in particular for the continuation of circular orbits [26]. Geometrically, it is simply a continuation of trajectories of Kepler problem into trajectories of the 3-body problem. It amounts to considering $\mu$ as a small parameter - the limit case $\mu=0$ being Kepler problem in the rotating frame- writing

$$
H_{0}=\frac{|p|^{2}}{2}-{ }^{t} q K p-\frac{1}{|q|}+o(\mu) .
$$

The approximation for $\mu$ is valid, a neighbourhood of the primaries being excluded. In the Earth-Moon problem, since $\mu$ is very small, the Kepler problem is a good approximation of the motion in a large neighbourhood of the Earth.

4.4.1. The control problem. The control system in the rotating frame is deduced from the previous model and can be written in Hamiltonian form

$$
\frac{\mathrm{d} x}{\mathrm{~d} t}=\vec{H}_{0}(x)+u_{1} \vec{H}_{1}(x)+u_{2} \vec{H}_{2}(x)
$$

where $x=(q, p), \vec{H}_{0}$ is the free motion and $\vec{H}_{1}, \vec{H}_{2}$ are given by $\vec{H}_{i}=-q_{i}, i=1,2$. As for the Kepler problem, the mass variation of the satellite can be introduced in the model dividing $u_{i}$ by $m(t)$ and adding the equation $\dot{m}=-\delta|u|$. Again, it will be not taken into account here. Moreover we still restrict our analysis to the energy minimization problem with fixed final time $t_{f}$ and control valued in $\mathbf{R}^{2}$. The physical problem which is to maximize the final mass can be analyzed using a continuation method. Preliminary results in the minimum time case are given in [15].

A lunar mission using low propulsion called SMART-1 was realized by ESA and the practical details of the mission - in particular the description of the trajectoryare reported in $[29,30]$. We present a trajectory analysis based on our geometric and numerical techniques. For simplicity, we have fixed the boundary conditions to circular orbits, the one around the Earth corresponding to the geostationary one. But everything can be applied to other boundary conditions, for instance those described in the report status of the SMART-1 mission. A trajectory comparison is discussed in the final section (see also [6]).

Our analysis is based on a numerical continuation, including second-order optimality check, where $\mu$ is the parameter of the continuation. The averaged system is finally applied to get an approximate energy minimizing trajectory for the phase of 
the mission that starts from the circular Earth orbit and aims at a quasi-elliptic orbit where the apogee is about 338000 Kilometers. In the restricted problem approximation, the effect of the inclination (around 30 degrees in phase two of the mission) is not modelled here.

4.4.2. Numerical continuation for the Earth- $\mathrm{L}_{2}$ transfer. As a first approach we choose to simulate the Earth- $L_{2}$ transfer in the restricted 3-body problem. Indeed, in the limit case $\mu=0$, the Moon and the point $L_{2}$ are identical. Moreover, in the Earth-Moon system, the point $L_{2}$ and the Moon are very close. As a result, the first phase of an Earth-Moon transfer is comparable to an Earth- $L_{2}$ transfer. Solving the shooting function associated with the Earth- $L_{2}$ transfer is consequently useful to provide a good approximation of the solution of the Earth-Moon transfer shooting function.

Using a circular orbit around the Earth for the geostationary one, we set as initial condition $x_{0}=(1-\mu+1.099 e-1,0,0,2.8792)$. The point $L_{2}$ is located on $\left(x_{L_{2}}, 0\right)$ with $x_{L_{2}}$ solution of the equation

$$
x-\frac{(1-\mu)(x+\mu)}{\rho_{1}^{3}}-\frac{\mu(x-1+\mu)}{\rho_{2}^{3}}=0 .
$$

Since we want to reach $L_{2}$ with a zero speed, we fix the target $x_{f}=\left(x_{L_{2}}, 0,0,0\right)$. By making the parameter $\mu$ vary from zero, one builds up a family $\left(S_{\mu}\right)_{\mu}$ of shooting functions which connects the Kepler and the 3-body problem. The numerical continuation method can be applied to deduce low thrust extremal trajectories of the Earth- $L_{2}$ transfer from the Kepler ones.

In accordance with the report status of ESA, we fix the transfer time to 121 time units of the restricted 3-body problem, which approximately corresponds to the transfer time from the Earth to the point $L_{2}$ during the SMART-1 mission (about 17 months). In addition, we consider a constant spacecraft mass of 350 Kilograms, see $[29,30]$. Setting $\mu=0$, we compute an extremal using the shooting method, then increase $\mu$ up to $1.2153 e-2$ with a discrete continuation. At each step, the first conjugate time along the extremal is computed to ensure convergence of the continuation method. The Euclidian norm of the extremal control is plotted Fig. 14 to draw a comparison between the control bound and the maximum thrust allowed by electro-ionic engines. Figs. 7 to 14 present the computed spacecraft trajectories in both rotating and fixed frames, as well as the first conjugate time and the norm of the control along trajectories in Kepler and Earth-Moon systems.

The numerical continuation method, considering $\mu$ as a small parameter, leads to deduce an extremal trajectory of the energy minimization Earth- $L_{2}$ transfer problem from one corresponding to the Kepler case, in accordance with Poincaré's work. The second order optimality condition check ensure that the computed extremals are locally energy minimizing in $\mathrm{L}^{\infty}\left(\left[0, t_{f}\right]\right)$. We also note that in both cases $\mu=0$ and 


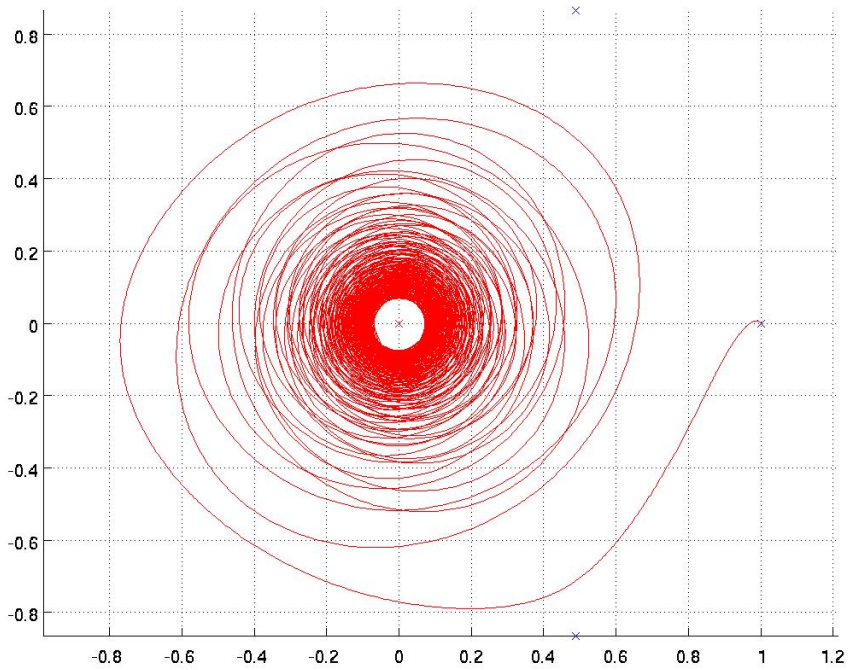

FIG. 7. Earth- $L_{2}$ trajectory in the rotating frame, $\mu=0$.

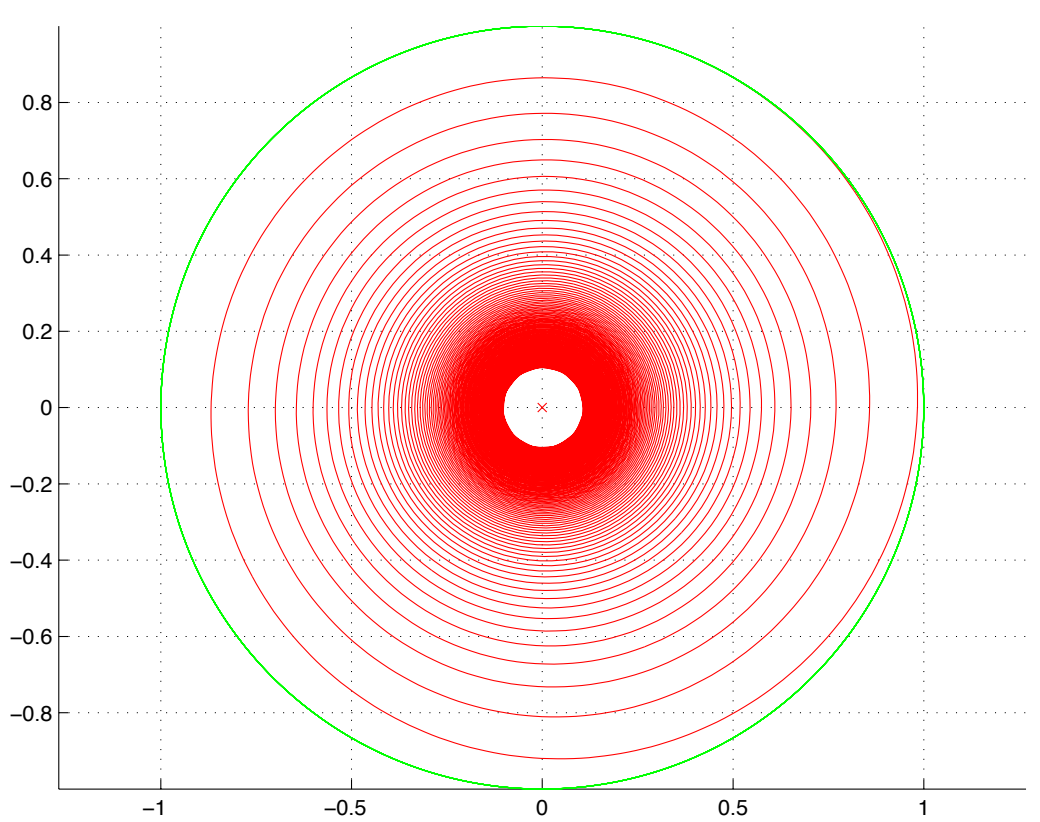

FIG. 8. Earth- $L_{2}$ trajectory in the fixed frame, $\mu=0$.

$\mu=1.2153 e-2$, the maximum value reached by the norm of the extremal control is inferior to the bound $|u| \leq 8 e-2$ of the SMART-1 electro-ionic engine $(7.3 e-2$ Newtons), while transfer times are comparable. 

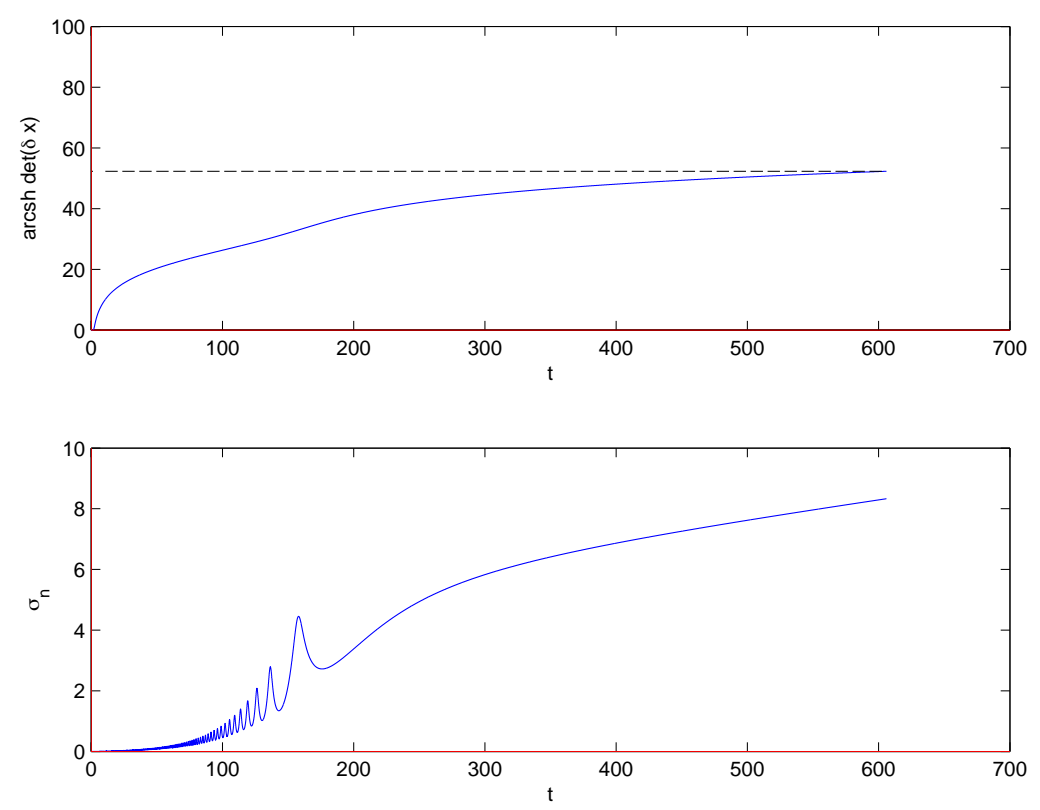

FIG. 9. Second order condition check. Neither the determinant of Jacobi fields nor the associated smallest singular value vanish, ensuring local optimality of the whole extremal.

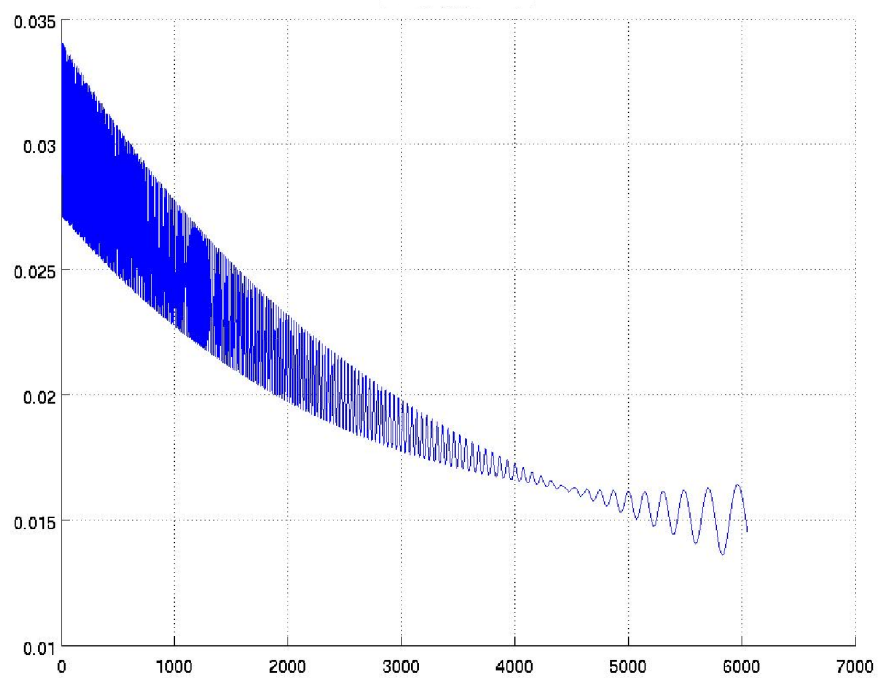

FIG. 10. Norm of the extremal control, Earth- $L_{2}$ transfer, $\mu=0$.

\subsubsection{Numerical continuation method for the Earth-Moon transfer.} The second part of our trajectory analysis is devoted to the Earth-Moon transfer. We use the same dynamics and initial conditions as previously. In this case, the circular 


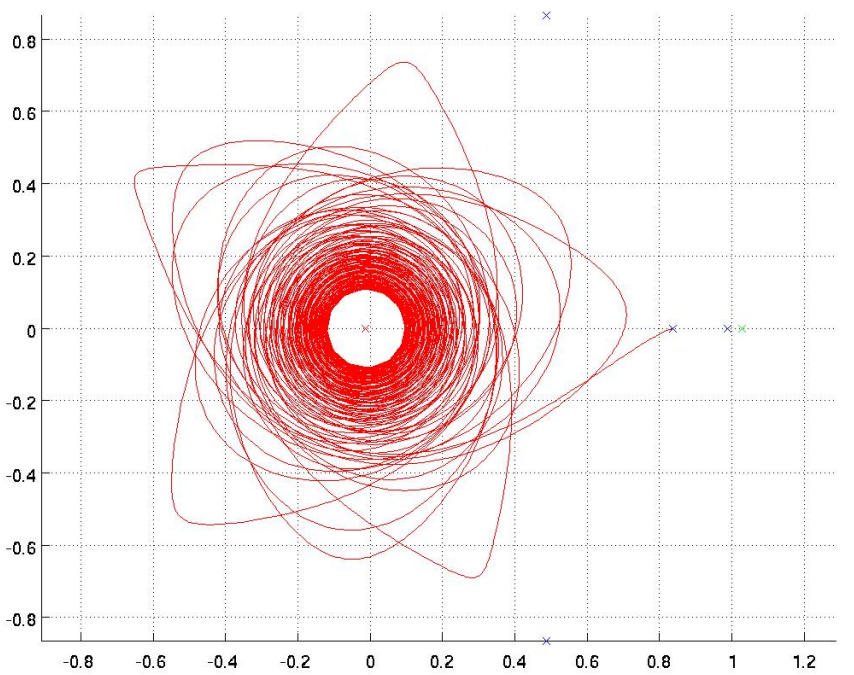

FIG. 11. Earth- $L_{2}$ trajectory in the rotating frame, $\mu=1.2153-2$.

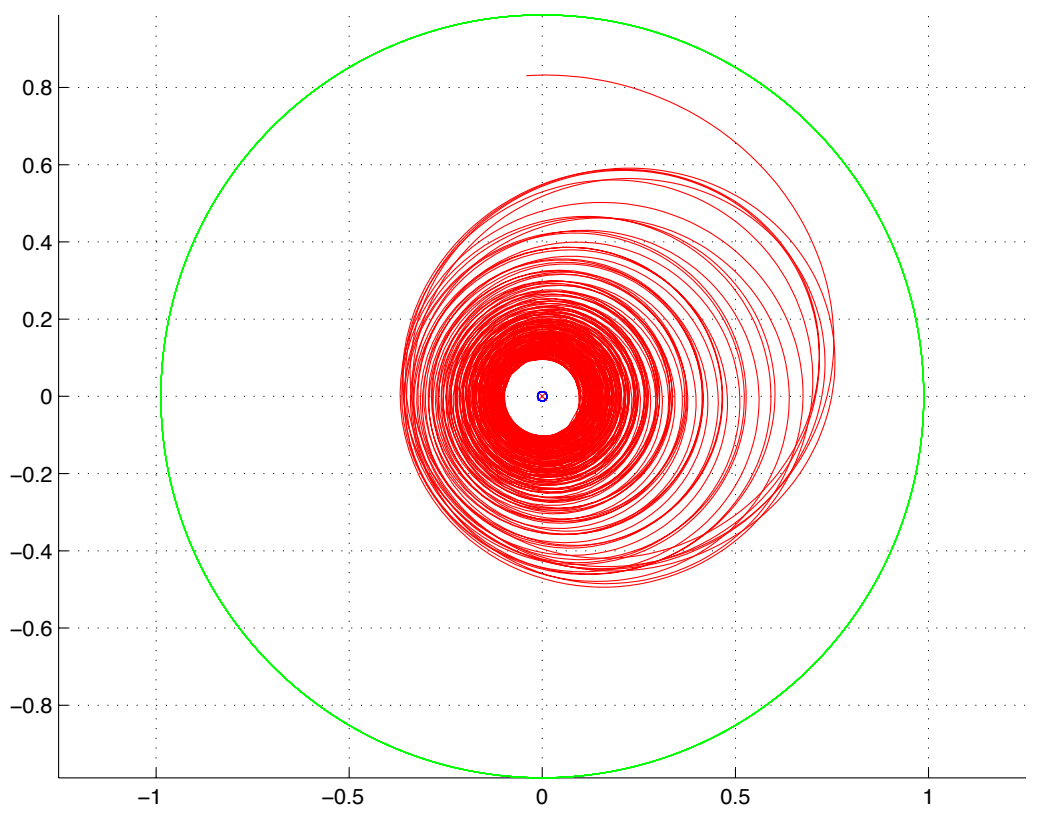

FIG. 12. Earth- $L_{2}$ trajectory in the fixed frame, $\mu=1.2153 e-2$.

orbit around the Moon, denoted $O_{L}$ and defined by

$$
\left(x_{1}-1+\mu\right)^{2}+x_{2}^{2}=1.7 e-3, \quad x_{3}^{2}+x_{4}^{2}=2.946 e-1, \quad\left(x_{1}-1+\mu, x_{2}\right) \perp\left(x_{3}, x_{4}\right),
$$



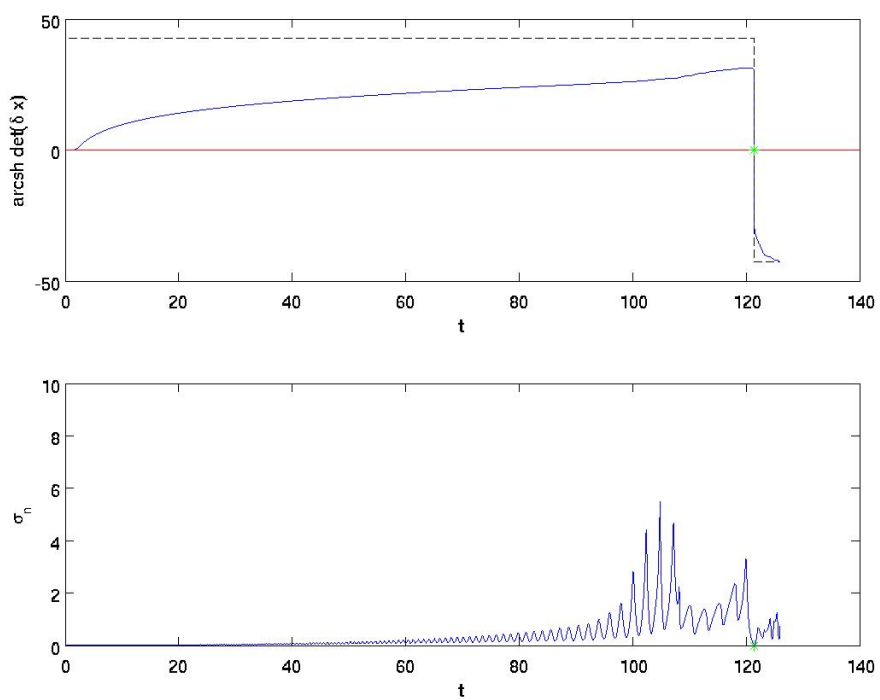

FIG. 13. First conjugate time, Earth- $L_{2}$ transfer, $\mu=1.2153 e-2$.

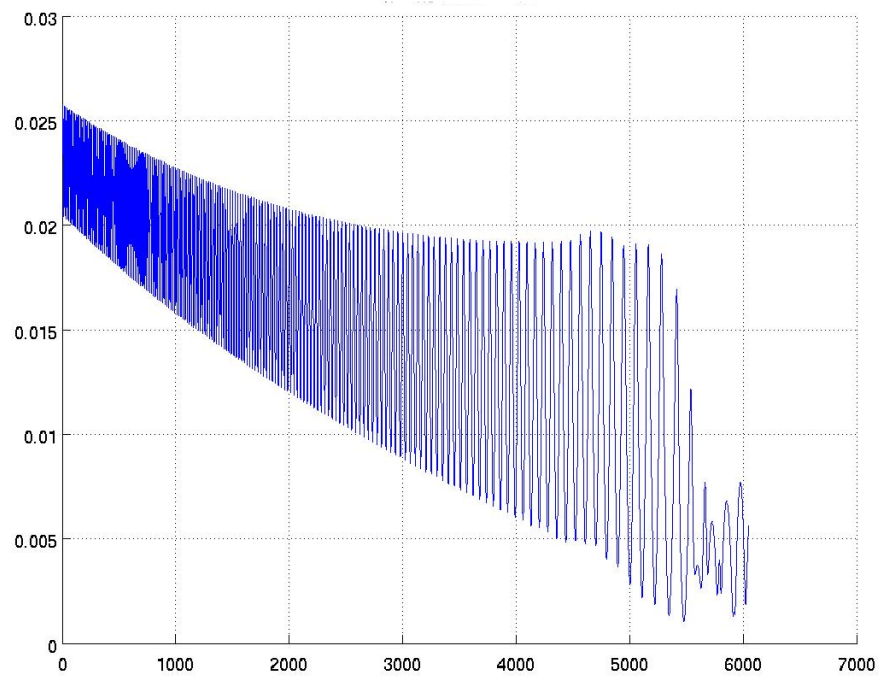

FIG. 14. Norm of the extremal control, Earth- $L_{2}$ transfer, $\mu=1.2153 e-2$.

is chosen as the target orbit. In addition to the former necessary conditions, the maximum principle provides the transversality condition $p\left(t_{f}\right) \perp T_{x\left(t_{f}\right)} O_{L}$. The shooting equation is modified accordingly, and one has to check local optimality computing focal instead of conjugate points.

The transfer time is fixed to 124 time units of the restricted 3-body problem. The 
extremal trajectory is obtained using the shooting method and initializing $p_{0}$ with the initial costate vector of the Earth- $L_{2}$ transfer. The Earth-Moon trajectories in both rotating and fixed frames, the first focal time and the norm of the extremal control are presented from Fig. 15 to Fig. 22 for $\mu$ varying between 0 and $1.2153 e-2$. In the two cases $\mu=0$ and $\mu=1.2153 e-2$, the first focal time along extremals $t_{1, \text { foc }}$ is higher than $3 t_{f} / 2$, ensuring local optimality. The maximal bound of the norm of the extremal control is about $4.5 e-2$ which approximately corresponds to the half of the maximal thrust allowed during SMART-1 mission.

It is interesting to notice that the Earth- $L_{2}$ Keplerian trajectory differs from the Earth-Moon Keplerian trajectory. The first phase of the Earth-Moon transfer matches the Earth- $L_{2}$ transfer. It underlines the crucial role of the neighbourhood of the point $L_{2}$ where the attractions of the two primaries compensate each other. By treating the first phase as a Keplerian transfer from the geostationary orbit (GEO) to a geostationary transfer orbit (GTO), we are able to use the averaging results of section 3.2 in order to estimate the maximal bound of the control associated with energy minimizing trajectories.

The first phase of the Earth-Moon trajectory with $\mu=1.2153 e-2$ can be approximated by a Keplerian transfer from the GEO to a GTO orbit with semi-major axis $a \simeq 5.84 e-1$, eccentricity $e \simeq 3.96 e-1$, and argument of pericenter $\theta=8 \pi / 7$. Using the system of coordinates introduced in Proposition 3.9, one can compute the appropriate geodesic and estimate the final longitude required to achieve a prescribed maximal bound on the norm of the control thanks to Proposition 3.7.

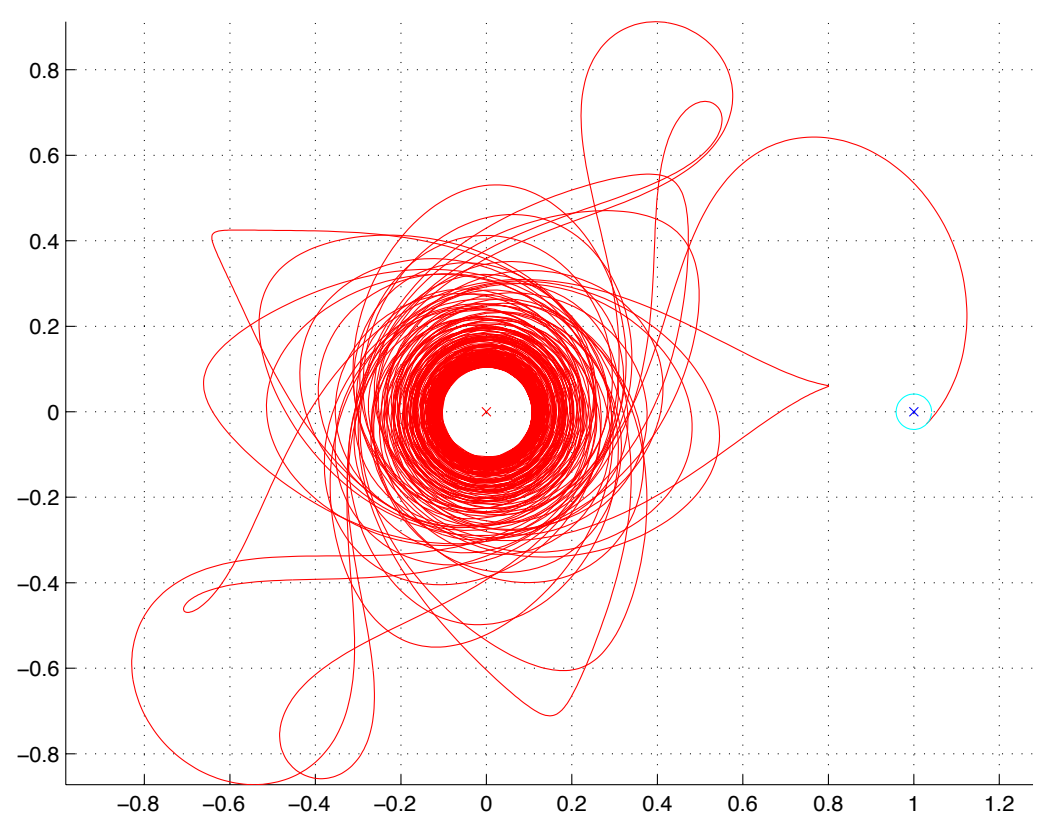

FIG. 15. Earth-Moon trajectory in the rotating frame, $\mu=0$. 


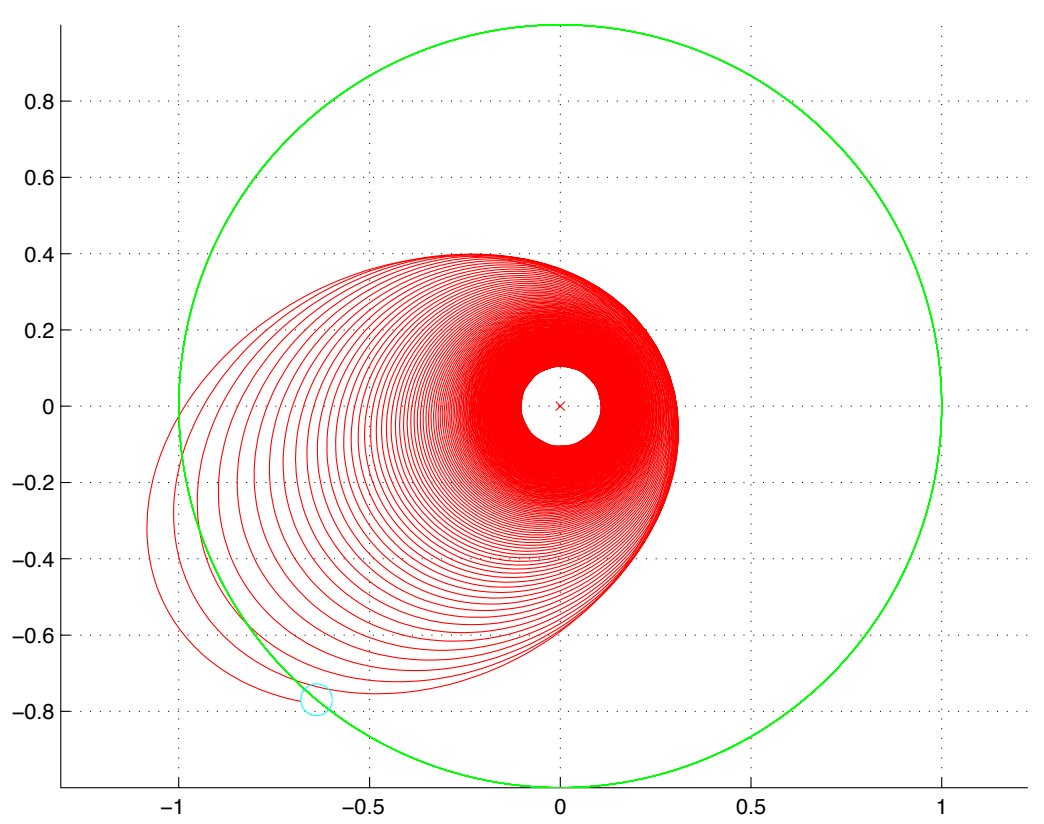

FIG. 16. Earth-Moon trajectory in the fixed frame, $\mu=0$.
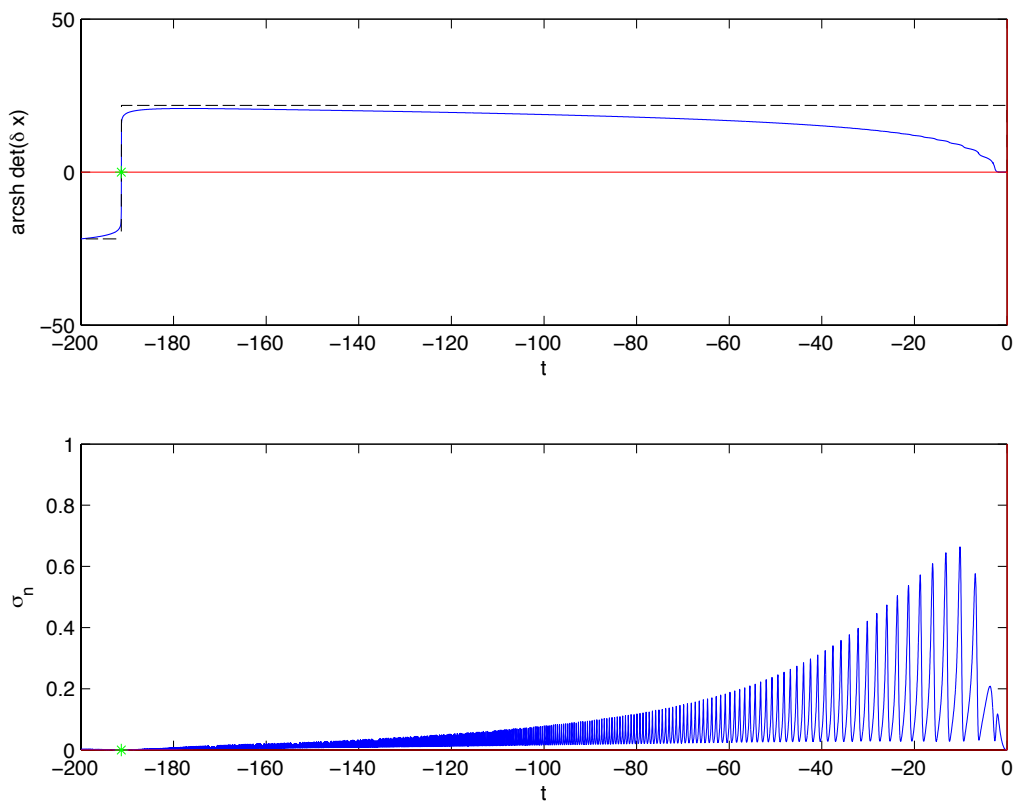

FIG. 17. First focal time and norm of extremal control, Earth-Moon transfer, $\mu=0$. 


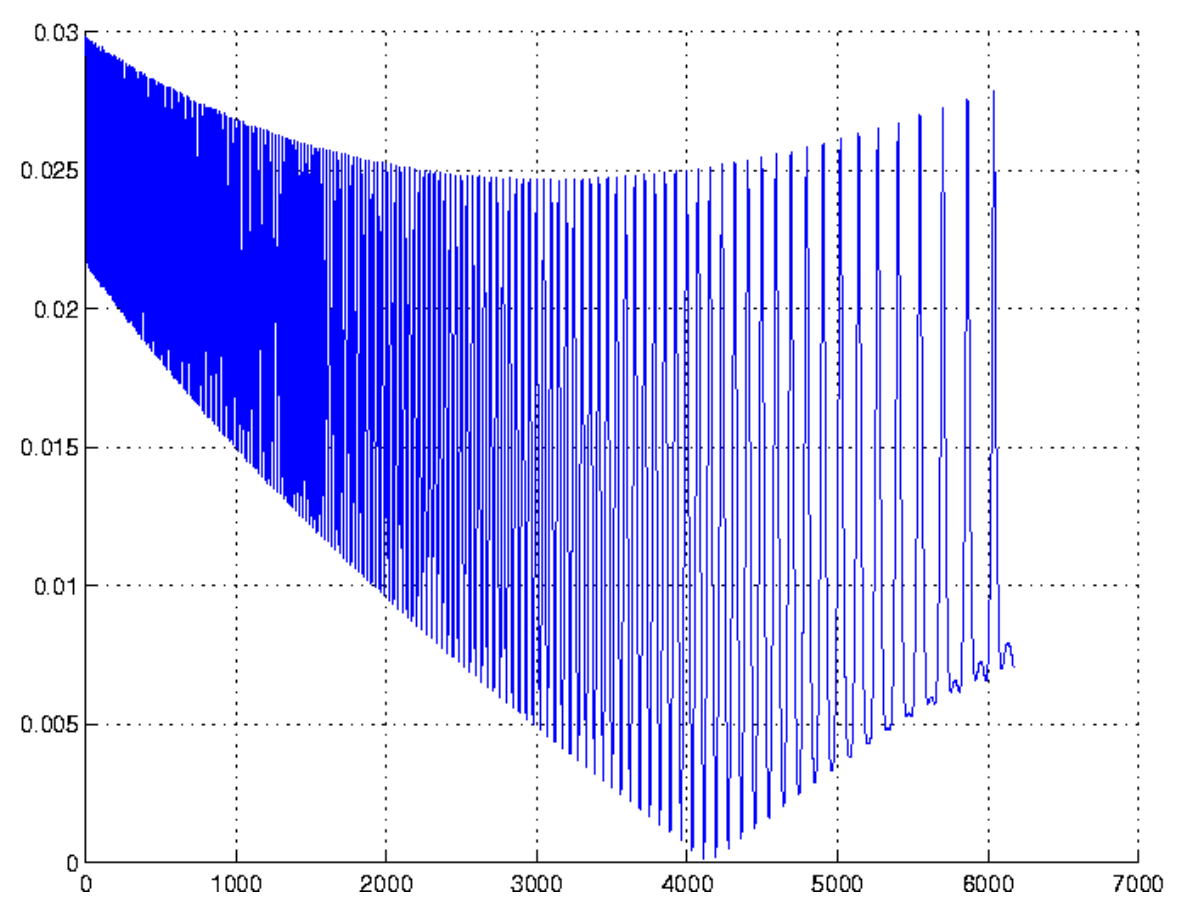

FIG. 18. Norm of extremal control, Earth-Moon transfer, $\mu=0$.

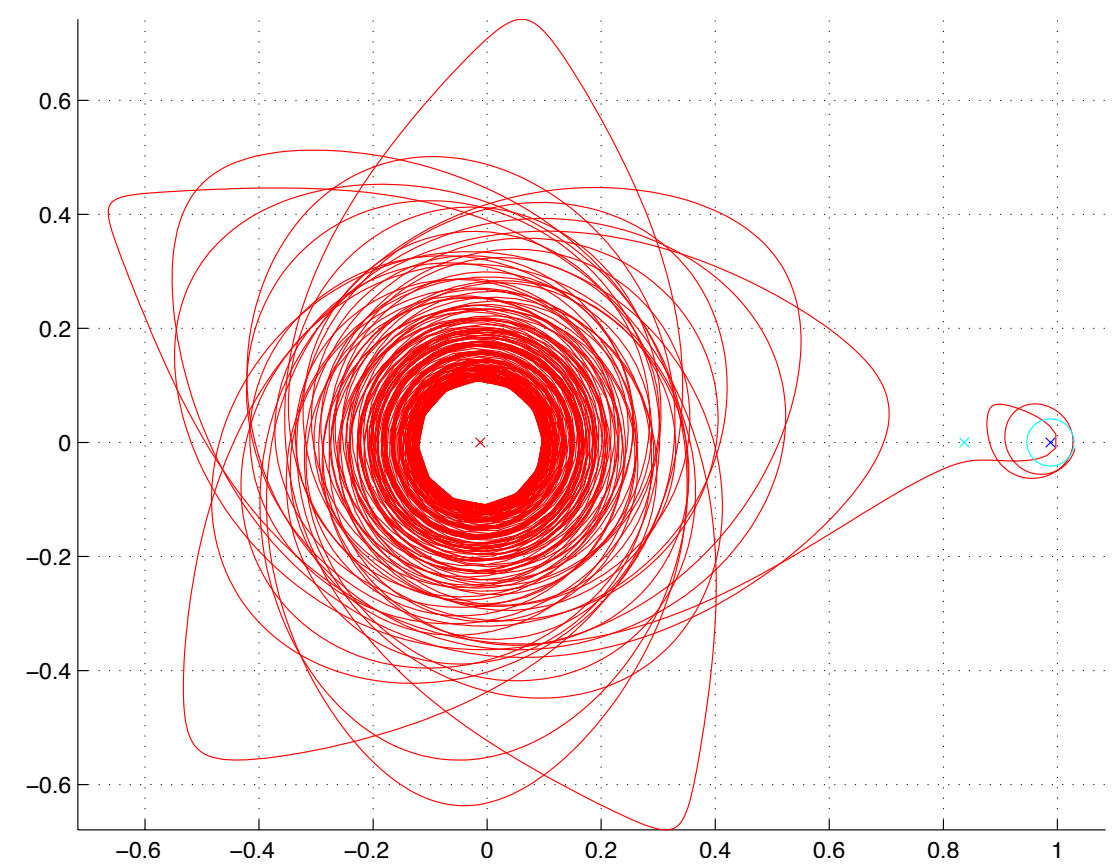

FIG. 19. Earth-Moon trajectory in the rotating frame, $\mu=1.2153 e-2$. 


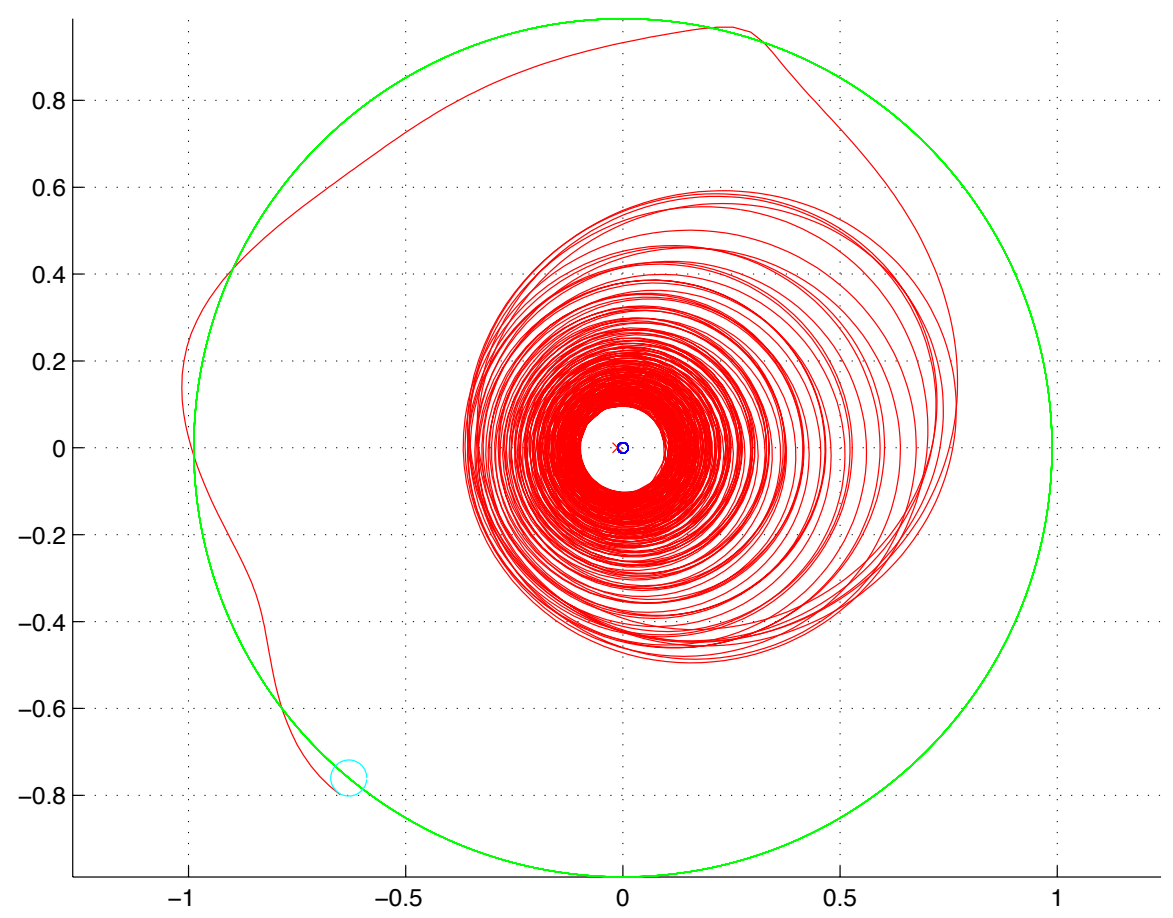

FIG. 20. Earth-Moon trajectory in the fixed frame, $\mu=1.2153 e-2$.
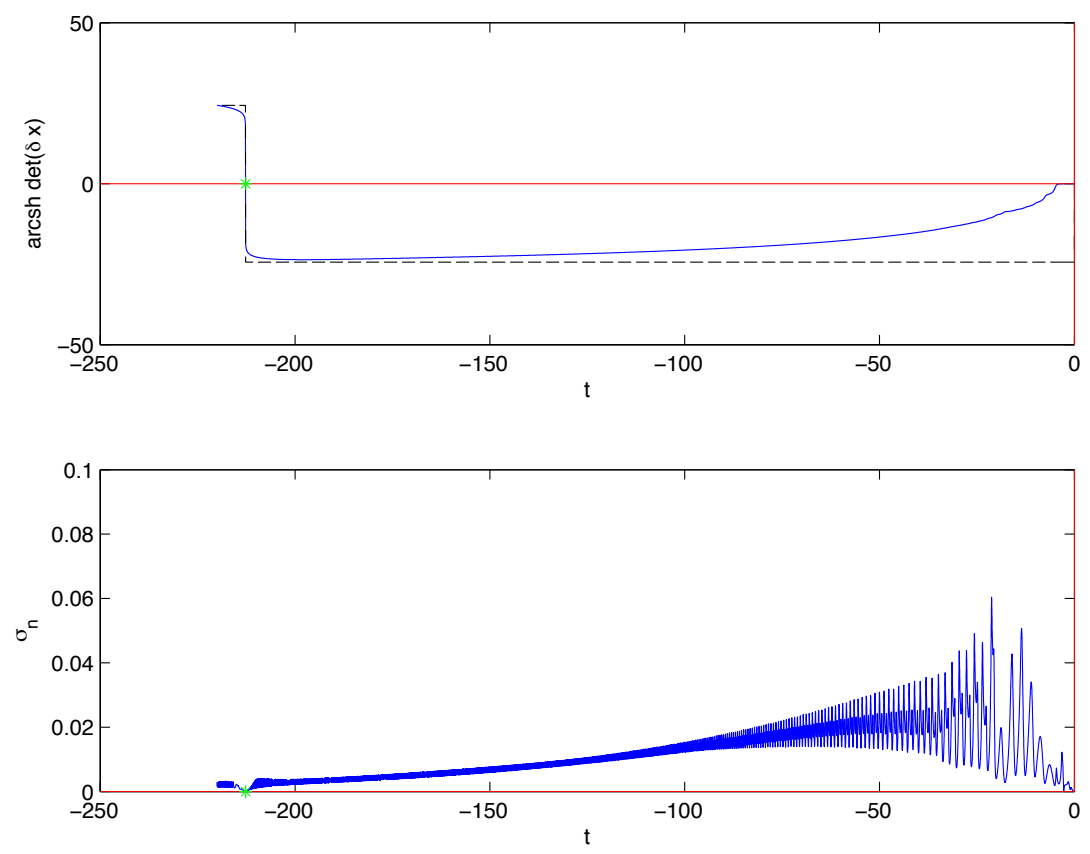

FiG. 21. First focal time, Earth-Moon transfer, $\mu=1.2153 e-2$. 


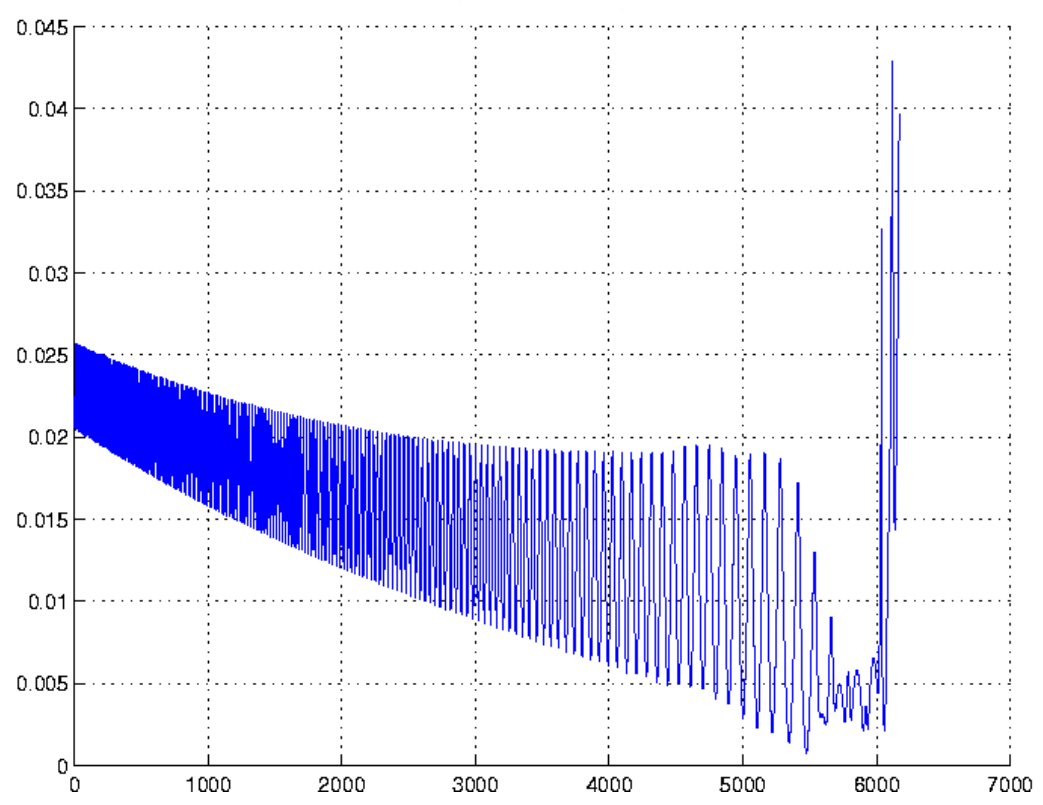

Fig. 22. Norm of extremal control, Earth-Moon transfer, $\mu=1.2153 e-2$.

\section{REFERENCES}

[1] E. L. Allgower And K. Georg, Numerical continuation methods, an introduction. Springer, Berlin, 1990.

[2] V. I. Arnold, Mathematical methods of classical mechanics. Springer, New-York, 1989.

[3] A. Bellaïche, The tangent space in sub-Riemannian geometry. Sub-Riemannian geometry, 1-78, Progr. Math. 144, Birkhäuser, 1996.

[4] A. Bensoussan, Regular perturbations in optimal control. Singular perturbations in systems and control (Udine, 1982), 169-183, CISM Courses and Lectures, 280, Springer, Vienna, 1983.

[5] G. A. Buiss, Lectures on the Calculus of Variations. Chicago, 1946.

[6] A. Bombrun, J. Chetboun, And J.-B. Pomet, Transfert Terre-Lune en poussée faible par contrôle feedback - La mission SMART-1. INRIA Research report (2006), no. 5955, 1-27.

[7] B. Bonnard AND J.-B. CaIllau, Riemannian metric of the averaged energy minimization problem in orbital transfer with low thrust. Ann. Inst. H. Poincaré Anal. Non Linéaire, 24:3(2007), pp. 395-411.

[8] B. Bonnard and J.-B. Caillau, Geodesic flow of the averaged controlled Kepler equation. Forum math. 21:5(2009), pp. 797-814.

[9] B. Bonnard and J.-B. Caillau, Singular metrics on the two-sphere in space mechanics. HAL preprint (2008), no. 00319299, pp. 1-25.

[10] B. Bonnard, J.-B. CAIllau, AND R. Dujol, Energy minimization of single-input orbit transfer by averaging and continuation. Bull. Sci. Math. 130:8(2006), pp. 707-719.

[11] B. Bonnard, J.-B. Caillau, R. Sinclair, and M. Tanaka, Conjugate and cut loci of a twosphere of revolution with application to optimal control. Ann. Inst. H. Poincaré Anal. Non Linéaire 26:4(2009), pp. 1081-1098.

[12] B. Bonnard, J.-B. Caillau, And E. Trélat, Second order optimality conditions in the smooth case and applications in optimal control. ESAIM Control Optim. and Calc. Var. 13:2(2007), 
pp. 207-236 (apo.enseeiht.fr/cotcot).

[13] B. Bonnard, N. Shcherbakova, And D. Sugny, The smooth continuation method in optimal control with an application to control systems. ESAIM Control Optim. and Calc. Var, DOI: $10.1051 / \mathrm{cocv} / 2010004$.

[14] J.-B. Caillau, Contribution à l'étude du contrôle en temps minimal des transferts orbitaux. Phd thesis, Toulouse Univ., 2000.

[15] J.-B. Caillau, B. Daoud, and J. Gergaud, On some Riemannian aspects of two and threebody controlled problems. Recent Advances in Optimization and its Applications in Engineering, Springer, to appear. Proceedings of the 14th Belgian-French-German Conference on Optimization, Leuven, September 2009.

[16] F. Chaplais, Averaging and deterministic optimal control. SIAM J. Control Optim. 25:3(1987), pp. $767-780$.

[17] M. P. Do CARMo, Riemannian geometry. Birkhäser, Boston, 1992.

[18] T. N. Edelbaum, Optimal low-thrust rendez-vous and station keeping. AIAA J. 2:7(1964), pp. 1196-1201.

[19] S. Geffroy, Généralisation des techniques de moyennation en contrôe optimal. Application aux problèems de transfert et rendez-vous orbitaux à poussée faible. Phd thesis, Toulouse Univ., 1997.

[20] J. Gergaud and T. Haberkorn, Homotopy method for minimum consumption orbit transfer problem. ESAIM Control Optim. Calc. Var. 12:2(2006), pp. 294-310.

[21] J. Guckenheimer And P. Holmes, Nonlinear oscillations, dynamical systems and bifurcations of vector fields. Springer, 1993.

[22] M. Guerra AND A. SARYChev, Existence and Lipschitzian regularity for relaxed minimizers. Mathematical control theory and finance, 231-250, Springer, Berlin, 2008.

[23] T. Kato, Perturbation theory for linear operators. Springer, 1980.

[24] J. E. Marsden And S. D. Ross, New methods in celestial mechanics and mission design, Bull. Amer. Math. Soc. (N.S.) 43:1(2006), pp. 43-73.

[25] K. Meyer AND G. R. HALL, Introduction to Hamiltonian dynamical systems and the N-body problem. Springer, New York, 1992.

[26] H. Poincaré, Oeuvres. Gauthier-Villars, Paris, 1934.

[27] H. Pollard, Mathematical introduction to celestial mechanics. Prentice-Hall, Englewood Cliffs, 1966.

[28] L. S. Pontryagin, V. G. Boltyanskit, R. V. Gamkrelidze, and E. F. Mishchenko, The mathematical theory of optimal processes. John Wiley \& Sons, New York, 1962.

[29] G. Racca, B. H. Foing, and M. Coradini, SMART-1: The first time of Europe to the Moon. Earth, Moon and planets 85-86(2001), pp. 379-390.

[30] G. RACCA ET AL. SMART-1 mission description and development status. Planetary and space science, 50(2002), pp. 1323-1337.

[31] R. Sinclair And M. TANAKA, The cut locus of a two-sphere of revolution and Toponogov's comparison theorem. Tohoku Math. J. 59:2(2007), pp. 379-399.

[32] V. Szebehely, Theory of orbits: The restricted problem of three bodies. Academic Press, 1967. 